
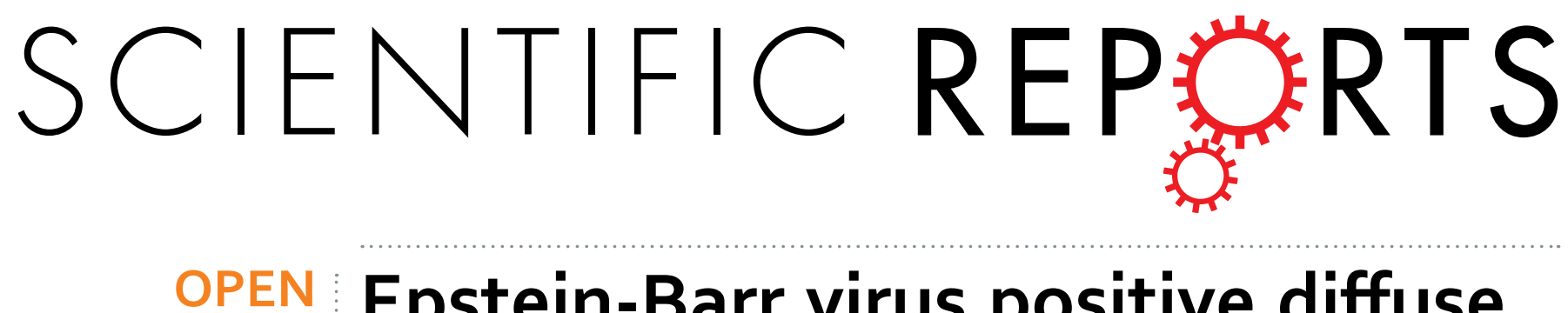

Received: 26 March 2015

Accepted: 16 June 2015

Published: 23 July 2015

\section{Epstein-Barr virus positive diffuse large B-cell lymphoma predict poor outcome, regardless of the age}

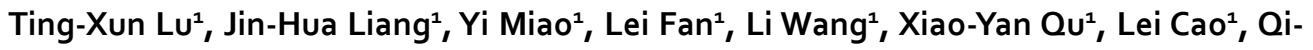
Xing Gong ${ }^{2}$, Zhen Wang ${ }^{2}$, Zhi-Hong Zhang ${ }^{2}$, Wei $\mathrm{Xu}^{1}$ \& Jian-Yong $\mathrm{Li}^{1}{ }^{1,3}$

Epstein-Barr virus (EBV) positive diffuse large B-cell lymphoma (DLBCL) of the elderly is defined as patients older than 50 years alone. However, recent studies showed young patients with sound immune status could also be affected. In this study, we investigated the clinical features and outcomes of patients with EBV positive DLBCL in the different age groups using different EBER cutoff values. The prevalence of EBV positive DLBCL was $14.0 \%$ (35/250) and 10.4\% (26/250) for EBER cut-off of $20 \%$ and $50 \%$, respectively. With both EBER cut-off values, patients with EBV DLBCL shared many unfavorable prognostic characteristics, regardless of age. EBV positive patients, both in the elderly and young groups, showed significantly worse overall survival and progression-free survival than negative cases. Moreover, no significant differences of outcomes were identified between different age groups with EBV positive DLBCL. In conclusion, EBV positive DLBCL patients, regardless of age, shared similar poor prognostic features and showed worse outcome than negative cases. We suggest that the age criterion of EBV positive DLBCL of the elderly, and possibly the name itself, be modified in future.

Diffuse large B-cell lymphoma (DLBCL) is the most common subtype of malignant lymphoma. DLBCL harboring Epstein-Barr virus (EBV) positive monoclonal B-cell proliferation in patients older than 50 years without any known immunodeficiency or prior lymphoma is termed EBV positive DLBCL of the elderly ${ }^{1,2}$. The EBV positive DLBCL of the elderly accounts for $8.7 \%-11.4 \%$ of all DLBCL in Asian countries $^{3-6}$, but less than $5 \%$ in western nations $s^{7,8}$. Since the introduction of rituximab, R-CHOP has become the standard treatment for CD20 positive DLBCL ${ }^{9,10}$. The outcome of DLBCL patients is improved with R-CHOP, but the impact on the prognosis of EBV positive DLBCL patients remains controversial ${ }^{5,6,11,12}$.

Most of studies showed the outcome of elderly patients with EBV positive DLBCL treated with R-CHOP was worse than negative ones, ${ }^{4,512-16}$. While few reports showed the impact of EBV positivity was overcome with R-CHOP especially patients received more than three cycles of therapies ${ }^{7,11}$.

Of note, recent reports demonstrated that EBV positive DLBCL could also affect younger patients ( $<50$ years), who also showed poor response to traditional immunochemotherapy ${ }^{4,16-19}$. Hong et al. ${ }^{14}$ showed the prevalence of EBV positivity in the young group was less frequent (young vs. elderly: 6.7\% vs. 9.3\%), compared with the elderly group. However, they found the EBV positivity in the young group was not closely associated with unfavorable clinical features which was restricted to the elderly group. In addition, the poor prognostic impact of EBV positivity on overall survival (OS) and progression-free survival (PFS) was not observed in the young group, but in the elderly group alone $e^{14}$.

However, almost all of the previous studies, to our knowledge, analyzed the poor prognostic impact in the elderly or young group alone. None of them compared the clinical characteristics and prognosis

${ }^{1}$ Department of Hematology, the First Affiliated Hospital of Nanjing Medical University, Jiangsu Province Hospital, Nanjing 210029, China. ${ }^{2}$ Department of Pathology, the First Affiliated Hospital of Nanjing Medical University, Jiangsu Province Hospital, Nanjing 210029, China. ${ }^{3}$ Collaborative Innovation Center for Cancer Personalized Medicine, Nanjing Medical University, Nanjing 210029, China. Correspondence and requests for materials should be addressed to W.X. (email: xuwei100oo@hotmail.com) or J.-Y.L. (email: lijianyonglm@medmail.com.cn) 
between the elderly and young groups. Whether EBV positive DLBCL should be divided into two clinically distinct disease entities is still unknown. In present study, we investigated the clinical features of patients with EBV positive DLBCL and the outcome in different age groups.

\section{Material and methods}

Ethics statement. All patients provided informed consent in accordance with requirements of the Declaration of Helsinki, and the research project was approved by the University and Institutional Review Boards.

Patients. According to the 2008 World Health Organization (WHO) classification, we reviewed the medical records of 250 patients who diagnosed as de novo DLBCL at our hospital between July 2006 and December 2014. Patients with unknown EBV status, primary central nervous system lymphoma, post-transplant lymphoproliferative disorders, primary mediastinal B-cell lymphoma, and HIV-positive DLBCL were excluded from the study. All of the patients were treated with rituximab plus chemotherapy or chemotherapy alone.

Epstein-Barr virus-encoded RNA (EBER) in situ hybridization. EBER in situ hybridization was carried out using a fluorescein-conjugated EBER oligonucleotide probe and the purified IgG fraction of a mouse monoclonal anti-fluorescein antibody. Both $20 \%$ and $50 \%$ were applied as cut-off values for EBER positive tumour cells to assess the differences in clinical parameters, pathological features and survival differences ${ }^{20}$.

Immunohistochemistry (IHC). Antibodies applied in the study, according to the manufacturer's instructions, included CD5 (clone EP2952, Abcam, cut-off: 30\%), CD10 (clone 56C6, Dako, cut-off: 30\%), CD30 (clone CON6D/B5, Abcam, cut-off: 30\%), Ki-67 (clone Mib-1, Dako), Myc (clone Y69, Abcam, cut-off: 40\%), Bcl2 (clone 124, Dako, cut-off: 50\%), Bcl6 (clone LN22, Dako, cut-off: 30\%), MUM1 (clone MUM1p, Dako, cut-off: 30\%), FOXP1 (clone JC12, Abcam, cut-off: 60\%), GCET1 (clone RAM341; Abcam, cut-off: 60\%) and LMO2 (clone 1A9-1, Santa Cruz, cut-off: 30\%). The cell of origin (COO) was classified according to Hans, Choi, Tally and Visco-Young algorithms. The specific cut-off of each antibody used in different algorithms was described previously ${ }^{21-24}$.

Fluorescence in situ hybridization (FISH). FISH analysis was performed according to the manufacturer's instructions with MYC dual-color, break-apart translocation probe (Vysis LSI) and IGH/BCL2 dual-color, dual-fusion translocation probe (Vysis LSI). The cut-off levels for the probes were established by evaluating the split signal distribution in samples of reactive lymphoid tissues, calculating the mean number of split signals plus three times the standard deviation. The cut-off levels were $14 \%$ and $5 \%$ for MYC break apart probe and IGH/BCL2 dual-color, dual-fusion translocation probe, respectively.

Statistical analyses. Statistical analyses were performed using SPSS software, version 20.0. Chi-square and Fisher exact tests were used to compare categorical variables . OS and PFS were defined according to Cheson $2014^{25}$. Survival curves were plotted by using Kaplan-Meier method and were compared by using log-rank test. For all the tests, a probability value of less than 0.05 (2-sided) was considered statistically significant.

\section{Results}

Prevalence of EBV positive DLBCL in the cohort. A total of 250 cases with DLBCL were included in the analysis as the whole cohort. Using $20 \%$ as cut-off, $14.0 \%(35 / 250)$ cases showed EBER positivity. The prevalence of EBER positivity were $15.1 \%(25 / 166)$ and $11.9 \%(10 / 84)$ in the elderly and young group, respectively. No significant difference of incidence was observed between the two groups $(P=0.497)$. When a cut-off of $50 \%$ was used for EBER positivity, the incidence of EBER positive cases was $10.4 \%$ (26/250). Accordingly, 11.4\% (19/166) and 8.3\% (7/84) were positive for EBER in the elderly and young groups, respectively. No significant difference of prevalence was observed between the two groups $(P=0.466)$.

EBV positivity and clinical features. In the whole cohort, compared with EBER negative cases, EBER positive (for both cut-off values) patients showed male predominance, advanced clinical stages (stage III/IV), poor performance status (ECOG PS status 2-4) and lower response to first-line treatment. (Table 1,2$)$. Using $20 \%$ as cut-off, EBV positivity was significantly associated with male sex $(76.0 \%$ vs. $53.9 \%)$, poor PS status ( $40 \%$ vs. $12.5 \%)$ and lower response to first-line treatment (64.0\% vs. $84.4 \%$ ), compared with negative cases. In the young group, EBER positivity was strongly associated with poor PS status (30.0\% vs. 5.8\%) and lower response to first-line treatment (70.0\% vs. $94.6 \%)$, With regard to other clinical features, although not statistically significant, EBV positive patients more frequently showed unfavorable characteristics compared with negative cases in both elderly and young groups (Table 1). However, no significant differences of these clinical characteristics were observed between different age groups. When we analyzed above parameters with a cut-off value of $50 \%$ for EBER, although fewer 


\begin{tabular}{|c|c|c|c|c|c|c|c|c|c|c|}
\hline \multirow[b]{3}{*}{ Variable } & \multirow{3}{*}{$\begin{array}{c}\text { Number. } \\
(\%)\end{array}$} & \multicolumn{2}{|c|}{ All patients $(\mathrm{N}=250)$} & \multirow[b]{3}{*}{$P$ value } & \multicolumn{2}{|c|}{ Elderly group } & \multirow{3}{*}{$\begin{array}{c}P \\
\text { value }\end{array}$} & \multicolumn{2}{|c|}{ Young group } & \multirow{3}{*}{$\begin{array}{c}P \\
\text { value }\end{array}$} \\
\hline & & EBV+ & EBV- & & EBV+ & EBV- & & EBV+ & EBV- & \\
\hline & & \multicolumn{2}{|c|}{ Number. (\%) } & & \multicolumn{2}{|c|}{ Number. (\%) } & & \multicolumn{2}{|c|}{ Number. (\%) } & \\
\hline Age & 250 & 35 & 215 & & 25 & 141 & & 10 & 74 & \\
\hline$\geq 50 y$ & $166(66.4)$ & $25(71.4)$ & $141(65.6)$ & 0.497 & 25 & 141 & NA & NA & NA & NA \\
\hline$<50 y$ & $84(33.6)$ & $10(28.6)$ & $74(34.4)$ & & NA & NA & & 10 & 74 & \\
\hline Sex & 250 & 35 & 215 & & 25 & 141 & & 10 & 74 & \\
\hline Male & $144(57.6)$ & $27(77.1)$ & $117(54.4)$ & 0.012 & $19(76.0)$ & $76(53.9)$ & 0.040 & $8(80.0)$ & $41(55.4)$ & 0.182 \\
\hline Female & $106(42.4)$ & $8(22.9)$ & $98(45.6)$ & & $6(24.0)$ & $65(46.1)$ & & $2(20.0)$ & $33(45.6)$ & \\
\hline Site & 250 & 35 & 215 & & 25 & 141 & & 10 & 74 & \\
\hline Extranodal & $106(41.6)$ & $20(57.1)$ & $86(40.0)$ & 0.057 & $15(60.0)$ & $62(44.0)$ & 0.139 & $5(50.0)$ & $24(32.4)$ & 0.303 \\
\hline Lymph node & $144(58.4)$ & $15(42.9)$ & $129(60.0)$ & & $10(40.0)$ & $79(56.0)$ & & $5(50.0)$ & $50(67.6)$ & \\
\hline Stage & 250 & 35 & 215 & & 25 & 141 & & 10 & 74 & \\
\hline I/II & $125(50.0)$ & $12(34.3)$ & $113(52.6)$ & 0.045 & $8(32.0)$ & $68(48.2)$ & 0.133 & $4(40.0)$ & $45(60.8)$ & 0.307 \\
\hline III/IV & $125(50.0)$ & $23(65.7)$ & $102(47.4)$ & & $17(68.0)$ & $73(51.8)$ & & $6(60.0)$ & $29(39.2)$ & \\
\hline ECOG PS & 238 & 25 & 213 & & 15 & 144 & & 10 & 69 & \\
\hline $0-1$ & $207(83.5)$ & $16(64.0)$ & $191(89.7)$ & 0.002 & $9(60.0)$ & $126(87.5)$ & 0.013 & $7(70.0)$ & $65(94.2)$ & 0.040 \\
\hline $2-4$ & $31(16.5)$ & $9(36.0)$ & $22(10.3)$ & & $6(40.0)$ & $18(12.5)$ & & $3(30.0)$ & $4(5.8)$ & \\
\hline ESI & 242 & 30 & 212 & & 20 & 139 & & 10 & 73 & \\
\hline $0-1$ & $194(80.2)$ & $20(66.7)$ & $174(82.1)$ & 0.048 & $13(65.0)$ & $112(80.6)$ & 0.143 & $7(70.0)$ & $62(84.9)$ & 0.361 \\
\hline$>1$ & $48(19.8)$ & $10(33.3)$ & $38(17.9)$ & & $7(35.0)$ & 27 (19.4) & & $3(30.0)$ & $11(15.1)$ & \\
\hline IPI & 237 & 29 & 208 & & 19 & 139 & & 10 & 69 & \\
\hline $0-2$ & $174(73.4)$ & $17(58.6)$ & $157(75.5)$ & 0.054 & $9(47.4)$ & $93(66.9)$ & 0.095 & $8(80.0)$ & $64(92.8)$ & 0.214 \\
\hline $3-5$ & $63(26.6)$ & $12(41.4)$ & $51(24.5)$ & & $10(52.6)$ & $46(33.1)$ & & $2(20.0)$ & $5(7.2)$ & \\
\hline Treatment & 250 & 35 & 215 & & 25 & 141 & & 10 & 74 & \\
\hline R-CHOP & $125(50.0)$ & $27(77.1)$ & 98 (45.6) & NA & $19(76.0)$ & $58(41.1)$ & NA & $8(80.0)$ & $40(54.1)$ & NA \\
\hline R-DA- EPOCH & $35(14.0)$ & $7(20.0)$ & $28(13.0)$ & NA & $5(20.0)$ & $23(16.3)$ & NA & $2(20.0)$ & $5(6.8)$ & NA \\
\hline CHOP & $90(36.0)$ & $1(2.9)$ & 89 (41.4) & NA & $1(4.0)$ & $60(42.6)$ & NA & $0(0.0)$ & $29(39.1)$ & NA \\
\hline Response & 250 & 35 & 215 & & 25 & 141 & & 10 & 74 & \\
\hline $\mathrm{CR}(\mathrm{u}) / \mathrm{PR}$ & $212(84.8)$ & $23(65.7)$ & $189(87.9)$ & 0.001 & $16(64.0)$ & $119(84.4)$ & 0.025 & $7(70.0)$ & $70(94.6)$ & 0.034 \\
\hline No response & $38(15.2)$ & $12(34.3)$ & $26(12.1)$ & & $9(36.0)$ & $22(15.6)$ & & $3(30.0)$ & $4(5.4)$ & \\
\hline
\end{tabular}

Table 1. Patients' characteristics in different groups (EBER cut-off: 20\%). Abbreviations: EBV: EpsteinBarr virus; ESI: extranodal sites involvement; ECOG PS: Eastern Cooperative Oncology Group Performance Status; IPI: International Prognostic Index; NCCN: National Comprehensive Cancer Network; Chemo: R-CHOP, rituximab, cyclophosphamide, doxorubicin, vincristine, and prednisone; R-DA-EPOCH: Doseadjusted EPOCH-R ([etoposide, prednisone, vincristine, cyclophosphamide, doxorubicin] + rituximab); $\mathrm{CHOP}$, cyclophosphamide, doxorubicin, vincristine, and prednisone; chemotherapy; CR(u): complete remission(unconfirmed); PR: partial remission.

factors with significant differences were observed, EBV positive cases still showed majority of unfavaorable clinical features compared with negative ones, regardless of ages (Table 2).

EBV positivity and clinical prognostic indicators. In the whole cohort, EBER positivity (for both cut-off values) was significantly associated with elevated serum C reactive protein (CRP) level, $\beta 2$ microglobulin $(\beta 2 \mathrm{M})$ level, CA125 level, erythrocyte sedimentation rate (ESR) and Ferritin level (Table 3,4). Using $20 \%$ as cut-off, in the elderly patients, compared with EBER negative cases, EBER positive ones more frequently had elevated serum CRP level (71.4\% vs. $45.2 \%)$, $\beta 2 \mathrm{M}$ level $(71.4 \%$ vs. 45.8\%), CA125 level (61.5\% vs. $29.3 \%$ ), ESR (92.9\% vs. $22.7 \%$ ) and Ferritin level (55.6\% vs. $29.3 \%)$. In the young group, EBER positivity was associated with all of above clinical prognositic factors (Table 3 ). Additionally, no significant differences of these prognostic features were observed between different age groups. When we analyzed those parameters with the $50 \%$ for cut-off of EBER, similar results were observed (Table 4). 


\begin{tabular}{|c|c|c|c|c|c|c|c|c|c|c|}
\hline \multirow[b]{3}{*}{ Variable } & \multirow{3}{*}{$\begin{array}{c}\text { Number. } \\
(\%)\end{array}$} & \multicolumn{2}{|c|}{ All patients $(\mathrm{N}=250)$} & \multirow{3}{*}{$\begin{array}{c}\mathbf{P} \\
\text { value }\end{array}$} & \multicolumn{2}{|c|}{ Elderly group } & \multirow{3}{*}{$\begin{array}{c}\mathbf{P} \\
\text { value }\end{array}$} & \multicolumn{2}{|c|}{ Young group } & \multirow{3}{*}{$\begin{array}{c}\mathbf{P} \\
\text { value }\end{array}$} \\
\hline & & EBV+ & EBV- & & EBV+ & EBV- & & EBV+ & EBV- & \\
\hline & & \multicolumn{2}{|c|}{ Number. (\%) } & & \multicolumn{2}{|c|}{ Number. (\%) } & & \multicolumn{2}{|c|}{ Number. (\%) } & \\
\hline Age & 250 & 26 & 224 & & 19 & 147 & & 7 & 77 & \\
\hline$>50 y$ & $166(66.4)$ & $19(73.1)$ & $147(65.6)$ & & $19(100)$ & $147(65.6)$ & NA & NA & NA & NA \\
\hline$\leq 50 y$ & $84(33.6)$ & $7(26.9)$ & $77(34.4)$ & & NA & NA & & $7(100)$ & $77(34.4)$ & \\
\hline Sex & 250 & 26 & 224 & & 19 & 147 & & 7 & 77 & \\
\hline Male & $144(57.6)$ & $21(80.8)$ & $123(54.9)$ & 0.012 & $15(78.9)$ & $80(54.4)$ & 0.042 & $6(85.7)$ & $43(55.8)$ & 0.230 \\
\hline Female & $106(42.4)$ & $5(19.2)$ & $101(45.1)$ & & $4(21.1)$ & $67(45.6)$ & & $1(14.3)$ & $34(44.2)$ & \\
\hline Site & 250 & 26 & 224 & & 19 & 147 & & 7 & 77 & \\
\hline Extranodal & $106(41.6)$ & $11(42.3)$ & $95(42.4)$ & 0.992 & $9(47.4)$ & $68(46.3)$ & 0.927 & $2(28.6)$ & $27(35.1)$ & 1.000 \\
\hline Lymph node & $144(58.4)$ & $15(57.7)$ & $129(57.6)$ & & $10(52.6)$ & $79(53.7)$ & & $5(71.4)$ & $50(64.9)$ & \\
\hline Stage & 250 & 26 & 224 & & 19 & 147 & & 7 & 77 & \\
\hline I/II & $125(50.0)$ & $6(23.1)$ & $119(53.1)$ & 0.004 & $4(21.1)$ & $72(49.0)$ & 0.021 & $2(28.6)$ & 47 (61.0) & 0.122 \\
\hline III/IV & $125(50.0)$ & $20(76.9)$ & $105(46.9)$ & & $15(78.9)$ & $75(51.0)$ & & $5(71.4)$ & $30(39.0)$ & \\
\hline ECOG PS & 238 & 20 & 218 & & 13 & 146 & & 7 & 72 & \\
\hline $0-1$ & $207(83.5)$ & $14(70.0)$ & $193(88.5)$ & 0.001 & $9(69.2)$ & $126(86.3)$ & 0.111 & $5(71.4)$ & 67 (93.1) & 0.115 \\
\hline $2-4$ & $31(16.5)$ & $6(30.0)$ & $25(11.5)$ & & $4(30.8)$ & $20(13.7)$ & & $2(28.6)$ & $5(6.9)$ & \\
\hline ESI & 242 & 21 & 221 & & 14 & 145 & & 7 & 76 & \\
\hline $0-1$ & $194(80.2)$ & $16(76.2)$ & $178(80.5)$ & 0.577 & $10(71.4)$ & $115(79.3)$ & 0.501 & $6(85.7)$ & $63(82.9)$ & 1.000 \\
\hline$>1$ & $48(19.8)$ & $5(23.8)$ & 43 (19.5) & & $4(28.6)$ & $30(20.7)$ & & $1(14.3)$ & $13(17.1)$ & \\
\hline IPI & 237 & 20 & 217 & & 13 & 145 & & 7 & 72 & \\
\hline $0-2$ & $174(73.4)$ & $11(55.0)$ & $163(75.1)$ & 0.051 & $6(46.2)$ & $96(66.2)$ & 0.224 & $5(71.4)$ & 67 (93.1) & 0.115 \\
\hline $3-5$ & $63(26.6)$ & $9(45.0)$ & $54(24.9)$ & & $7(53.8)$ & $49(33.8)$ & & $2(28.6)$ & $5(6.9)$ & \\
\hline Treatment & 250 & 26 & 224 & & 19 & 147 & & 7 & 77 & \\
\hline R-CHOP & $125(50.0)$ & $23(88.5)$ & $102(45.6)$ & NA & $18(94.7)$ & $59(40.1)$ & NA & $5(71.4)$ & $43(55.8)$ & NA \\
\hline R-DA- EPOCH & $35(14.0)$ & $2(7.6)$ & $33(14.7)$ & NA & $0(0.0)$ & $28(19.1)$ & NA & $2(28.6)$ & $5(6.5)$ & NA \\
\hline CHOP & $90(36.0)$ & $1(2.9)$ & $89(39.7)$ & NA & $1(5.3)$ & $60(40.8)$ & NA & $0(0.0)$ & $29(37.7)$ & NA \\
\hline Response & 250 & 26 & 224 & & 19 & 147 & & 7 & 77 & \\
\hline $\mathrm{CR}(\mathrm{u}) / \mathrm{PR}$ & $212(84.8)$ & $18(69.2)$ & $194(86.6)$ & 0.037 & $13(68.4)$ & $135(91.8)$ & 0.008 & $5(71.4)$ & $73(94.8)$ & 0.076 \\
\hline No response & $38(15.2)$ & $8(30.8)$ & $30(13.4)$ & & $6(31.6)$ & $12(8.2)$ & & $2(28.6)$ & $4(5.2)$ & \\
\hline
\end{tabular}

Table 2. Patients' characteristics of in different groups (EBER cut-off: 50\%). Abbreviations: EBV: Epstein-Barr virus; ESI: extranodal sites involvement; ECOG PS: Eastern Cooperative Oncology Group Performance Status; IPI: International Prognostic Index; NCCN: National Comprehensive Cancer Network; Chemo: R-CHOP, rituximab, cyclophosphamide, doxorubicin, vincristine, and prednisone; R-DA-EPOCH: Dose-adjusted EPOCH-R ([etoposide, prednisone, vincristine, cyclophosphamide, doxorubicin] + rituximab); CHOP, cyclophosphamide, doxorubicin, vincristine, and prednisone; chemotherapy; $\mathrm{CR}(\mathrm{u})$ : complete remission(unconfirmed); PR: partial remission.

EBV positivity and pathological characteristics. In the whole cohort, EBER positivity (for both cut-off values) was significantly associated with less frequent Bcl6 expression, more common CD30 expression, higher Ki-67 expression $(\geq 70 \%)(P<0.001)$, and higher incidence of double hit lymphoma (DHL) (Table 5,6). Using 20\% as cut-off, EBER positivity was associated with less frequent Bcl2 $(40.0 \%$ vs. $62.1 \%)$, Bcl6 (28\% vs. $65 \%)$ and FOXP1 expression (45.5\% vs. $68.5 \%)$, more common CD30 (28.0\% vs. 6.4\%), higher Ki-67 expression (70.8\% vs. $34.0 \%)$ and Myc expression (60.9\% vs. 37.6\%), more incidence of double protein expression (DPE) (44.0\% vs. $24.8 \%)$, MYC gene rearrangement $(34.4 \%$ vs. $10.7 \%)$ and double hit lymphoma (DHL) (13.0\% vs. 1.0\%). Other factors, including: lower CD10 expression (16.0\% vs. $24.8 \%)$ and more common of non-GCB subtype with Choi $(72.7 \%$ vs. $57.9 \%)$ and Visco-Young (75.0\% vs. 59.3\%) algorithms although not statistically significant, were shared by EBV positive patients, compared with negative cases (Table 5). In the young group, EBER positivity was associated with lower CD10 (0\% vs. $28.0 \%)$ and $\mathrm{LMO} 2$ expression (60.0\% vs. $86.1 \%)$, more common with CD30 expression (30.0\% vs. $6.8 \%)$, MYC gene rearrangement (33.3\% vs. $10.8 \%)$ and BCL2/IGH translocation (33.3\% vs. $8.1 \%$ ) (Table 5). In addition, no significantly differences were recognized among all of the pathological 


\begin{tabular}{|c|c|c|c|c|c|c|c|c|c|c|}
\hline \multirow[b]{3}{*}{ Variable } & \multirow{3}{*}{$\begin{array}{c}\text { Number. } \\
(\%)\end{array}$} & \multicolumn{2}{|c|}{ All age } & \multirow[b]{3}{*}{$P$ value } & \multicolumn{2}{|c|}{ Elderly group } & \multirow[b]{3}{*}{$P$ value } & \multicolumn{2}{|c|}{ Young group } & \multirow{3}{*}{$\begin{array}{c}P \\
\text { value }\end{array}$} \\
\hline & & EBV+ & EBV- & & EBV+ & EBV- & & EBV+ & EBV- & \\
\hline & & \multicolumn{2}{|c|}{ Number. (\%) } & & \multicolumn{2}{|c|}{ Number. (\%) } & & \multicolumn{2}{|c|}{ Number. (\%) } & \\
\hline CRP & 117 & 20 & 97 & & 14 & 62 & & 6 & 35 & \\
\hline Over ULN & $57(48.7)$ & $15(75.0)$ & $42(43.3)$ & 0.010 & $10(71.4)$ & $28(45.2)$ & 0.076 & $5(83.3)$ & $14(40.0)$ & 0.080 \\
\hline Normal & $60(51.3)$ & $5(25.0)$ & $55(56.7)$ & & $4(28.6)$ & $34(54.8)$ & & $1(16.7)$ & $21(60.0)$ & \\
\hline$\beta 2 \mathrm{MG}$ & 134 & 22 & 109 & & 14 & 72 & & 8 & 40 & \\
\hline Over ULN & $59(44.0)$ & 16() & $43(72.7)$ & 0.003 & $10(71.4)$ & $33(45.8)$ & 0.080 & $6(75.0)$ & $10(25.0)$ & 0.012 \\
\hline Normal & $75(56.0)$ & 6() & $69(27.3)$ & & $4(28.6)$ & $39(54.2)$ & & $2(25.0)$ & $30(75.0)$ & \\
\hline TK1 & 92 & 15 & 77 & & 9 & 52 & & 6 & 25 & \\
\hline Over ULN & $28(30.4)$ & $4(26.7)$ & $24(31.2)$ & 1.000 & $2(22.2)$ & $15(28.8)$ & 1.000 & $2(33.3)$ & $9(36.0)$ & 1.000 \\
\hline Normal & $64(69.6)$ & $11(73.3)$ & $53(68.8)$ & & $7(77.8)$ & $37(71.2)$ & & $4(66.7)$ & $16(64.0)$ & \\
\hline CA125 & 108 & 18 & 90 & & 13 & 58 & & 5 & 32 & \\
\hline Over ULN & $42(38.9)$ & $13(72.2)$ & $29(32.2)$ & 0.001 & $8(61.5)$ & $17(29.3)$ & 0.051 & $5(100.0)$ & $12(37.5)$ & 0.014 \\
\hline Normal & $66(61.1)$ & $5(27.8)$ & $61(67.8)$ & & $5(38.5)$ & $41(70.7)$ & & $0(0.0)$ & $20(62.5)$ & \\
\hline ESR & 163 & 22 & 141 & & 14 & 88 & & 8 & 53 & \\
\hline Over ULN & $49(30.1)$ & $19(86.4)$ & $30(21.3)$ & $<0.001$ & $13(92.9)$ & $20(22.7)$ & $<0.001$ & $6(75.0)$ & $10(18.9)$ & 0.003 \\
\hline Normal & $114(69.9)$ & $3(13.6)$ & $111(78.7)$ & & $1(7.1)$ & $68(77.3)$ & & $2(25.0)$ & $43(81.1)$ & \\
\hline Ferritin & 152 & 24 & 128 & & 18 & 82 & & 6 & 46 & \\
\hline Over ULN & $52(34.2)$ & $15(62.5)$ & 37 (28.9) & 0.001 & $10(55.6)$ & $24(29.3)$ & 0.033 & $5(83.3)$ & $13(28.3)$ & 0.015 \\
\hline Normal & $100(65.8)$ & $9(37.5)$ & $91(71.1)$ & & $8(44.4)$ & $58(70.7)$ & & $1(16.7)$ & $33(71.7)$ & \\
\hline LDH & 250 & 34 & 216 & & 24 & 139 & & 10 & 77 & \\
\hline Over ULN & $114(45.6)$ & $20(58.8)$ & 94 (43.5) & 0.096 & $15(62.5)$ & $59(42.4)$ & 0.068 & $5(50.0)$ & 35 (45.5) & 1.000 \\
\hline Normal & $136(54.4)$ & $14(41.2)$ & $122(56.5)$ & & $9(37.5)$ & 80 (57.6) & & $5(50.0)$ & $42(54.5)$ & \\
\hline CK & 149 & 24 & 125 & & 16 & 70 & & 8 & 55 & \\
\hline Complex & $5(3.4)$ & $1(4.2)$ & $4(3.2)$ & 0.590 & $1(6.2)$ & $2(2.9)$ & 0.465 & $0(0.0)$ & $2(3.6)$ & 1.000 \\
\hline Normal & $144(96.6)$ & $23(95.8)$ & $121(96.8)$ & & 15 (93.8) & 68 (97.1) & & $8(100.0)$ & $53(96.4)$ & \\
\hline $\mathrm{BM}$ & 150 & 25 & 125 & & 17 & 81 & & 8 & 44 & \\
\hline Positive & $17(11.3)$ & $4(16.0)$ & $13(10.4)$ & 0.487 & $3(17.6)$ & $8(9.9)$ & 0.398 & $1(12.5)$ & $5(11.4)$ & 1.000 \\
\hline Negative & $133(88.7)$ & $21(84.0)$ & $112(89.6)$ & & $14(82.4)$ & $73(90.1)$ & & $7(87.5)$ & 39 (88.6) & \\
\hline
\end{tabular}

Table 3. Clinical features of patients in different groups (EBER cut-off: 20\%). Abbreviations: EBV: Epstein-Barr virus; CRP: serum C reactive protein; ULN: upper limit of normal; $\beta 2 \mathrm{MG:} \beta 2$ microglobulin; ESR: erythrocyte sedimentation rate; LDH: lactate dehydrogenase; $\mathrm{CK}$ : chromosome karyotype; BM: bone marrow.

features between different age groups. When we analyzed those parameters with the cut-off value of $50 \%$, similar results were observed in both age groups (Table 6).

Survival analysis. Prognosis of EBV status. In the whole cohort, after a median follow-up of 29.3 (range, 1.3-122.4) months, patients with EBV positive DLBCL showed significantly worse OS (median OS: $20 \%$ as EBER cut-off: 18.3 months vs. not reached, $P<0.0001 ; 50 \%$ as EBER cut-off: 37.0 months vs. not reached, $P=0.0021$ ) (Fig. 1a,c) and PFS (median PFS: 20\% as EBER cut-off: 11.9 months vs. not reached, $P<0.0001 ; 50 \%$ as EBER cut-off: 20.5 months vs. not reached, $P<0.0001)$ (Fig. 1b,d) than EBV negative ones.

Prognosis of EBV status in the elderly group. We carried out survival analysis in the elderly group. Patients with EBV positive DLBCL showed significantly worse OS (median OS: $20 \%$ as EBER cut-off: 17.0 months vs. not reached, $P<0.0001 ; 50 \%$ as EBER cut-off: 37.0 months vs. not reached, $P=0.0337$ ) (Fig. 2a,c) and PFS (median PFS: $20 \%$ as EBER cut-off: 9.8 months vs. not reached, $P<0.0001 ; 50 \%$ as EBER cut-off: 20.7 months vs. not reached, $P<0.0001$ ) (Fig. 2b,d) compared with those with EBV negative DLBCL.

Prognosis of EBV status in the young group. We carried out survival analysis in the young group. Patients with EBV positive DLBCL showed significantly worse OS (median OS: $20 \%$ as EBER cut-off: 32.2 months vs. not reached, $P<0.0001 ; 50 \%$ as EBER cut-off: 36.5 months vs. not reached, $P=0.0255)$ (Fig. 3a,c) and 


\begin{tabular}{|c|c|c|c|c|c|c|c|c|c|c|}
\hline \multirow[b]{3}{*}{ Variable } & \multirow{3}{*}{$\begin{array}{c}\text { Number. } \\
(\%)\end{array}$} & \multicolumn{2}{|c|}{ All age } & \multirow[b]{3}{*}{$P$ value } & \multicolumn{2}{|c|}{ Elderly group } & \multirow[b]{3}{*}{$P$ value } & \multicolumn{2}{|c|}{ Young group } & \multirow{3}{*}{$\begin{array}{c}P \\
\text { value }\end{array}$} \\
\hline & & EBV+ & EBV- & & EBV+ & EBV- & & EBV+ & EBV- & \\
\hline & & \multicolumn{2}{|c|}{ Number. (\%) } & & \multicolumn{2}{|c|}{ Number. (\%) } & & \multicolumn{2}{|c|}{ Number. (\%) } & \\
\hline CRP & 117 & 19 & 98 & & 13 & 63 & & 6 & 35 & \\
\hline Over ULN & $57(48.7)$ & $15(78.9)$ & $42(42.9)$ & 0.004 & $10(76.9)$ & $28(44.4)$ & 0.033 & $5(83.3)$ & $14(66.7)$ & 0.080 \\
\hline Normal & $60(51.3)$ & $4(21.1)$ & $56(57.1)$ & & $3(23.1)$ & 35 (55.6) & & $1(16.7)$ & $21(33.3)$ & \\
\hline$\beta 2 \mathrm{MG}$ & 134 & 18 & 116 & & 11 & 75 & & 7 & 41 & \\
\hline Over ULN & $59(44.0)$ & $14(77.8)$ & $45(38.8)$ & 0.002 & $9(81.8)$ & $34(45.3)$ & 0.024 & $5(71.4)$ & $11(26.8)$ & 0.033 \\
\hline Normal & $75(56.0)$ & $4(22.2)$ & $71(61.2)$ & & $2(18.2)$ & 41 (54.7) & & $2(28.6)$ & $30(73.2)$ & \\
\hline TK1 & 92 & 14 & 78 & & 8 & 53 & & 6 & 25 & \\
\hline Over ULN & $28(30.4)$ & $4(28.6)$ & $24(30.8)$ & 1.000 & $2(25.0)$ & $15(28.3)$ & 1.000 & $2(33.3)$ & $9(36.0)$ & 1.000 \\
\hline Normal & $64(69.6)$ & $10(71.4)$ & $54(69.2)$ & & $6(75.0)$ & $38(71.7)$ & & $4(66.7)$ & $16(64.0)$ & \\
\hline CA125 & 108 & 16 & 92 & & 11 & 60 & & 5 & 32 & \\
\hline Over ULN & 42() & $12(75.0)$ & $30(32.6)$ & 0.001 & $7(63.6)$ & $18(30.0)$ & 0.043 & $5(100.0)$ & $12(37.5)$ & 0.014 \\
\hline Normal & 66() & $4(25.0)$ & $62(67.4)$ & & $4(36.4)$ & $42(70.0)$ & & $0(0.0)$ & $20(62.5)$ & \\
\hline ESR & 163 & 21 & 142 & & 14 & 88 & & 7 & 54 & \\
\hline Over ULN & $49(30.1)$ & $18(85.7)$ & $31(21.8)$ & $<0.001$ & $13(92.9)$ & $20(22.7)$ & $<0.001$ & $5(71.4)$ & $11(20.4)$ & 0.011 \\
\hline Normal & $114(69.9)$ & $3(14.3)$ & $111(78.2)$ & & $1(7.1)$ & $68(77.3)$ & & $2(28.6)$ & $43(79.6)$ & \\
\hline Ferritin & 152 & 21 & 131 & & 16 & 84 & & 5 & 47 & \\
\hline Over ULN & $52(34.2)$ & $14(66.7)$ & $38(29.0)$ & 0.001 & $10(62.5)$ & 24 (28.6) & 0.009 & $4(80.0)$ & $14(29.8)$ & 0.043 \\
\hline Normal & $100(65.8)$ & $7(33.3)$ & $93(71.0)$ & & $6(37.5)$ & $60(71.4)$ & & $1(20.0)$ & $33(70.2)$ & \\
\hline LDH & 250 & 25 & 225 & & 18 & 145 & & 7 & 80 & \\
\hline Over ULN & $114(45.6)$ & $14(56.0)$ & $100(44.4)$ & 0.271 & $10(55.5)$ & $64(44.1)$ & 0.359 & $4(57.1)$ & $36(45.0)$ & 0.698 \\
\hline Normal & $136(54.4)$ & $11(44.0)$ & $125(55.6)$ & & $8(45.5)$ & 81 (55.9) & & $3(42.9)$ & $44(55.0)$ & \\
\hline CK & 149 & 21 & 128 & & 14 & 72 & & 7 & 56 & \\
\hline Complex & $5(3.4)$ & $1(4.8)$ & $4(3.1)$ & 0.537 & $1(7.1)$ & $2(27.8)$ & 0.417 & $0(0.0)$ & $2(3.6)$ & 1.000 \\
\hline Normal & $144(96.6)$ & $20(95.2)$ & $124(96.9)$ & & $13(92.9)$ & 70 (97.2) & & $7(100.0)$ & $54(96.4)$ & \\
\hline $\mathrm{BM}$ & 150 & 22 & 128 & & 15 & 83 & & 7 & 45 & \\
\hline Positive & $17(11.3)$ & $4(18.2)$ & $13(10.2)$ & 0.279 & $3(20.0)$ & $8(9.6)$ & 0.366 & $1(14.3)$ & $5(11.1)$ & 1.000 \\
\hline Negative & $133(88.7)$ & $18(81.8)$ & $115(89.8)$ & & $12(80.0)$ & $75(90.4)$ & & $6(85.7)$ & $40(88.9)$ & \\
\hline
\end{tabular}

Table 4. Clinical features of patients in different groups (EBER cut-off: 50\%). Abbreviations: EBV: Epstein-Barr virus; CRP: serum C reactive protein; ULN: upper limit of normal; $\beta 2 \mathrm{MG}$ : $\beta 2$ microglobulin; ESR: erythrocyte sedimentation rate; LDH: lactate dehydrogenase; CK: chromosome karyotype; BM: bone marrow.

PFS (median PFS: $20 \%$ as EBER cut-off: 16.5 months vs. not reached, $P<0.0001 ; 50 \%$ as EBER cut-off: 20.5 months vs. not reached, $P=0.0010$ ) (Fig. $3 c, d$ ) than patients with EBV negative DLBCL.

Prognosis of EBV positivity between different age groups. We further compared the survival difference between the elderly and young group. Unexpectedly, elderly patients with EBV positive DLBCL of the elderly group showed OS (median OS: $20 \%$ as EBER cut-off: 17.0 vs. 32.2 months, $P=0.8434 ; 50 \%$ as EBER cut-off: 37.0 vs. 36.5 months, $P=0.8058$ ) (Fig. $4 a, c)$ and PFS (median PFS: $20 \%$ as EBER cut-off: 9.8 vs. 16.3 months, $P=0.5878$; $50 \%$ as EBER cut-off: 20.7 vs. 20.5 months, $P=0.8323$ ) similar to those of their young counterparts (Fig. $4 \mathrm{~b}, \mathrm{~d}$ ). In addition, we also analyzed the survival differences with other age cut-offs (40 or 60 years old), however, no difference of OS or PFS was recognized between different age groups with EBV positive DLBCL (data not shown).

\section{Discussion}

While using 20\% and 50\% as cut-off vaues, the incidences of EBV positive DLBCL were $14.0 \%(35 / 250)$ and $10.4 \%(26 / 250)$, respectively. Our result was similar to that of a previous study in Peru ( $14.9 \%$ for $20 \%$ cut-off and $9.0 \%$ for $50 \%$ cut-off $)^{26}$. However, taking different cut-off vaues used in previous studies into consideration, the incidence of EBV positive lymphoma appeared to be higher than those reported in previous studies in Asian countries ${ }^{4,20}$. 


\begin{tabular}{|c|c|c|c|c|c|c|c|c|c|c|}
\hline \multirow[b]{3}{*}{ Variable } & \multirow{3}{*}{$\begin{array}{c}\text { Number. } \\
(\%)\end{array}$} & \multicolumn{2}{|c|}{ All age } & \multirow[b]{3}{*}{$P$ value } & \multicolumn{2}{|c|}{ Elderly group } & \multirow{3}{*}{$\begin{array}{c}P \\
\text { value }\end{array}$} & \multicolumn{2}{|c|}{ Young group } & \multirow{3}{*}{$\begin{array}{c}P \\
\text { value }\end{array}$} \\
\hline & & EBV+ & EBV- & & EBV+ & EBV- & & EBV+ & EBV- & \\
\hline & & \multicolumn{2}{|c|}{ Number.(\%) } & & \multicolumn{2}{|c|}{ Number.(\%) } & & \multicolumn{2}{|c|}{ Number.(\%) } & \\
\hline CD10 & 250 & 35 & 215 & & 25 & 141 & & 10 & 74 & \\
\hline Positive & $60(24.0)$ & $4(11.4)$ & $56(26.0)$ & 0.060 & $4(16.0)$ & $35(24.8)$ & 0.338 & $0(0.0)$ & $21(28.4)$ & 0.060 \\
\hline Negative & $190(76.0)$ & 31 (88.6) & $159(74.0)$ & & $21(84.0)$ & $106(75.2)$ & & $10(100)$ & $53(71.6)$ & \\
\hline Bcl6 & 248 & 35 & 223 & & 25 & 140 & & 10 & 73 & \\
\hline Positive & $160(64.5)$ & $12(34.3)$ & $148(66.4)$ & $<0.001$ & $7(28.0)$ & $91(65.0)$ & 0.001 & $5(50.0)$ & $57(78.1)$ & 0.113 \\
\hline Negative & 88 (35.5) & $23(65.7)$ & 65 (33.6) & & $18(72.0)$ & $49(35.0)$ & & $5(50.0)$ & $16(21.9)$ & \\
\hline MUM1 & 250 & 35 & 215 & & 25 & 141 & & 10 & 74 & \\
\hline Positive & $157(62.8)$ & $23(65.7)$ & $134(62.3)$ & 0.700 & $16(64.0)$ & $95(67.4)$ & 0.741 & $7(70.0)$ & $39(52.7)$ & 0.500 \\
\hline Negative & $93(37.2)$ & $12(34.3)$ & $81(37.7)$ & & $9(36.0)$ & $46(32.6)$ & & $3(30.0)$ & $35(47.3)$ & \\
\hline GCET1 & 245 & 32 & 213 & & 22 & 141 & & 10 & 72 & \\
\hline$\geq 60 \%$ & $31(12.7)$ & $3(9.4)$ & $28(13.1)$ & 0.776 & $2(9.1)$ & $18(12.8)$ & 1.000 & $1(10.0)$ & $10(13.9)$ & 1.000 \\
\hline$<60 \%$ & $214(87.3)$ & $29(90.6)$ & $185(86.9)$ & & $20(90.9)$ & $123(87.2)$ & & $9(90.0)$ & $62(86.1)$ & \\
\hline FOXP1 & 230 & 32 & 198 & & 22 & 130 & & 10 & 68 & \\
\hline$\geq 60 \%$ & $138(60.1)$ & $17(53.1)$ & $121(61.1)$ & 0.392 & $10(45.5)$ & 89 (68.5) & 0.036 & $7(70.0)$ & $32(47.1)$ & 0.176 \\
\hline$<60 \%$ & $92(35.9)$ & $15(46.9)$ & 77 (38.9) & & $12(54.5)$ & $41(31.5)$ & & $3(30.0)$ & $36(52.9)$ & \\
\hline $\mathrm{LMO} 2$ & 244 & 32 & 222 & & 22 & 140 & & 10 & 72 & \\
\hline$\geq 30 \%$ & $194(79.5)$ & 25 (78.1) & $169(76.1)$ & 0.439 & $19(86.4)$ & $107(76.4)$ & 0.412 & $6(60.0)$ & $62(86.1)$ & 0.062 \\
\hline$<30 \%$ & $50(20.5)$ & $7(21.9)$ & $33(23.9)$ & & $3(13.6)$ & $33(23.6)$ & & $4(40.0)$ & $10(13.9)$ & \\
\hline CD5 & 250 & 35 & 215 & & 25 & 141 & & 10 & 74 & \\
\hline Positive & $17(6.8)$ & $2(5.7)$ & $15(7.0)$ & 1.000 & $1(4.0)$ & $7(5.0)$ & 1.000 & $1(10.0)$ & $8(10.8)$ & 1.000 \\
\hline Negative & $233(93.2)$ & $33(94.3)$ & $200(93.0)$ & & $24(96.0)$ & $134(95.0)$ & & $9(90.0)$ & 66 (89.2) & \\
\hline CD30 & 250 & 35 & 215 & & 25 & 141 & & 10 & 74 & \\
\hline Positive & $25(10.0)$ & $10(28.6)$ & $15(6.5)$ & 0.001 & $7(28.0)$ & $9(6.4)$ & 0.003 & $3(30.0)$ & $6(6.8)$ & 0.070 \\
\hline Negative & $225(90.0)$ & $25(71.4)$ & $200(93.4)$ & & $18(72.0)$ & $132(93.6)$ & & $7(70.0)$ & 68 (93.2) & \\
\hline Myc & 248 & 33 & 215 & & 23 & 141 & & 10 & 74 & \\
\hline Positive & 91 (36.7) & $19(57.6)$ & $72(33.5)$ & 0.008 & $14(60.9)$ & $53(37.6)$ & 0.035 & $5(50.0)$ & 19 (25.7) & 0.140 \\
\hline Negative & $157(63.3)$ & $14(42.4)$ & $143(66.5)$ & & $9(39.1)$ & $88(62.4)$ & & $5(50.0)$ & $55(74.3)$ & \\
\hline $\mathrm{Bcl} 2$ & 249 & 35 & 214 & & 25 & 140 & & 10 & 74 & \\
\hline Positive & $142(57.0)$ & $14(40.0)$ & $128(59.8)$ & 0.028 & $10(40.0)$ & $87(62.1)$ & 0.038 & $4(40.0)$ & $41(55.4)$ & 0.503 \\
\hline Negative & $107(43.0)$ & $21(60.0)$ & $86(40.2)$ & & $15(60.0)$ & $53(37.9)$ & & $6(60.0)$ & $33(44.6)$ & \\
\hline DPE & $64(25.6)$ & $15(42.9)$ & 49 (22.8) & 0.012 & $11(44.0)$ & $35(24.8)$ & 0.048 & $4(40.0)$ & 14 (18.9) & 0.210 \\
\hline Others & $186(74.4)$ & $20(57.1)$ & $166(77.2)$ & & $14(56.0)$ & $106(75.2)$ & & $6(60.0)$ & $60(81.1)$ & \\
\hline Ki-67 & 249 & 34 & 215 & & 24 & 141 & & 10 & 74 & \\
\hline$\geq 70 \%$ & $97(39.0)$ & $23(67.6)$ & $74(34.4)$ & $<0.001$ & $17(70.8)$ & $48(34.0)$ & 0.001 & $6(60.0)$ & $26(35.1)$ & 0.170 \\
\hline$<70 \%$ & $152(61.0)$ & $11(23.4)$ & $141(65.6)$ & & $7(29.2)$ & $93(66.0)$ & & $4(40.0)$ & 48 (64.9) & \\
\hline MYC-ba & 246 & 32 & 214 & & 23 & 140 & & 9 & 74 & \\
\hline Positive & $33(13.4)$ & $10(31.2)$ & $23(10.7)$ & 0.004 & $7(34.4)$ & $15(10.7)$ & 0.018 & $3(33.3)$ & $8(10.8)$ & 0.094 \\
\hline Negative & $213(86.6)$ & $22(68.8)$ & $191(89.3)$ & & $16(69.6)$ & 125 & & $6(66.7)$ & 66 (89.2) & \\
\hline BCL2/IGH & 247 & 32 & 215 & & 23 & 141 & & 9 & 74 & \\
\hline Positive & $32(13.0)$ & $7(21.9)$ & 25 (11.6) & 0.152 & $4(17.4)$ & $19(13.5)$ & 0.535 & $3(33.3)$ & $6(8.1)$ & 0.054 \\
\hline Negative & $215(87.0)$ & $25(78.1)$ & $190(88.4)$ & & $19(82.6)$ & $122(86.5)$ & & $6(66.7)$ & 68 (91.9) & \\
\hline DHL & $6(2.4)$ & $4(12.1)$ & $2(1.0)$ & 0.003 & $3(13.0)$ & $1(1.0)$ & 0.009 & $1(10.0)$ & $1(1.4)$ & 0.225 \\
\hline Non-DHL & $242(97.6)$ & $29(87.9)$ & $213(99.0)$ & & $20(87.0)$ & $140(99.0)$ & & $9(90.0)$ & 73 (98.6) & \\
\hline Hans & 250 & 35 & 215 & & 25 & 141 & & 10 & 74 & \\
\hline GCB & $98(39.2)$ & $14(40.0)$ & $84(39.1)$ & 0.917 & $9(36.0)$ & 49 (34.8) & 0.904 & $5(50.0)$ & $35(47.3)$ & 1.000 \\
\hline Non-GCB & $152(60.8)$ & $21(60.0)$ & $131(60.9)$ & & $16(64.0)$ & $92(65.2)$ & & $5(50.0)$ & $39(52.7)$ & \\
\hline
\end{tabular}




\begin{tabular}{|c|c|c|c|c|c|c|c|c|c|c|}
\hline \multirow[b]{3}{*}{ Variable } & \multirow{3}{*}{$\begin{array}{c}\text { Number. } \\
(\%)\end{array}$} & \multicolumn{2}{|c|}{ All age } & \multirow[b]{3}{*}{$P$ value } & \multicolumn{2}{|c|}{ Elderly group } & \multirow{3}{*}{$\begin{array}{c}P \\
\text { value }\end{array}$} & \multicolumn{2}{|c|}{ Young group } & \multirow{3}{*}{$\begin{array}{c}P \\
\text { value }\end{array}$} \\
\hline & & EBV+ & EBV- & & EBV+ & EBV- & & EBV+ & EBV- & \\
\hline & & \multicolumn{2}{|c|}{ Number.(\%) } & & \multicolumn{2}{|c|}{ Number.(\%) } & & \multicolumn{2}{|c|}{ Number.(\%) } & \\
\hline Choi & 244 & 23 & 221 & & 22 & 140 & & 10 & 72 & \\
\hline GCB & $110(45.1)$ & $10(43.5)$ & $100(45.2)$ & 0.092 & $6(27.3)$ & $59(42.1)$ & 0.186 & $4(40.0)$ & $41(56.9)$ & 0.335 \\
\hline Non-GCB & $134(54.9)$ & $22(56.5)$ & $112(54.8)$ & & $16(72.7)$ & $81(57.9)$ & & $6(60.0)$ & $31(43.1)$ & \\
\hline Tally & 246 & 32 & 214 & & 22 & 141 & & 10 & 73 & \\
\hline GCB & $68(27.6)$ & $11(34.4)$ & $57(26.6)$ & 0.361 & $7(31.8)$ & $35(24.8)$ & 0.485 & $4(40.0)$ & $22(30.1)$ & 0.717 \\
\hline Non-GCB & $178(72.4)$ & $21(65.6)$ & $157(73.4)$ & & $15(68.2)$ & $106(75.2)$ & & $6(60.0)$ & $51(69.9)$ & \\
\hline Visco-Young & 246 & 34 & 212 & & 24 & 140 & & 10 & 72 & \\
\hline GCB & $106(43.1)$ & $11(32.4)$ & 95 (44.8) & 0.173 & $6(25.0)$ & $57(40.7)$ & 0.144 & $5(50.0)$ & $38(52.8)$ & 1.000 \\
\hline Non-GCB & $140(56.9)$ & $23(67.6)$ & $117(55.2)$ & & $18(75.0)$ & $83(59.3)$ & & $5(50.0)$ & $34(47.2)$ & \\
\hline
\end{tabular}

Table 5. Pathological features of patients in different groups (EBER cut-off: 20\%). Abbreviations: EBV: Epstein-Barr virus; DPE: double protein expression; ba: break apart; DHL: double hit lymphoma; GCB:germinal center B cell.
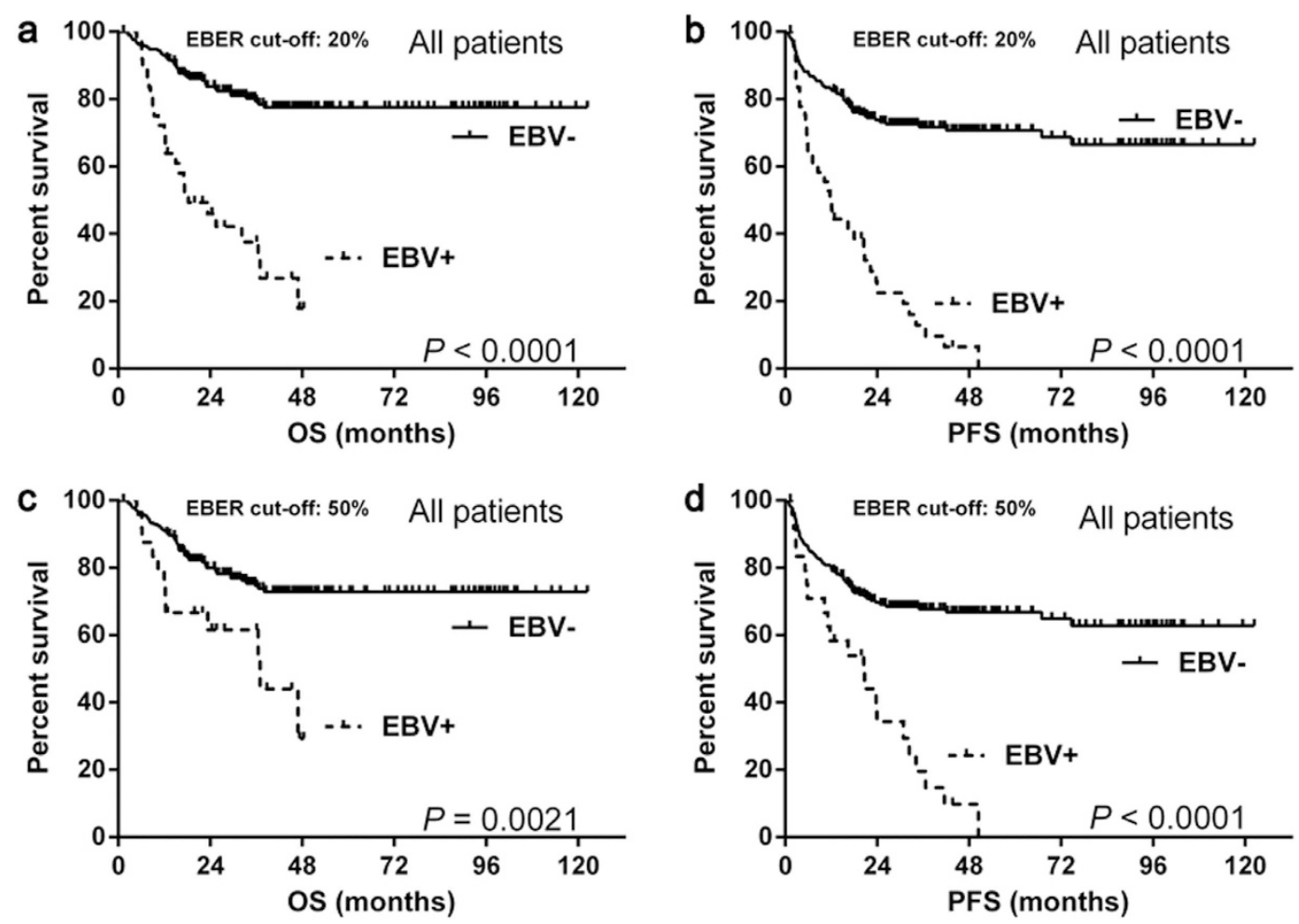

Figure 1. The overall survival and progession-free survival of the whole cohort. The EBV positive patients had significantly worse OS (a,c) and PFS (b,d) than the negative ones with both EBER cut-off values. Abbreviations: EBER: Epstein-Barr virus-encoded RNA; EBV: Epstein-Barr virus; OS: overall survival; PFS: progression-free survival.

In current study, EBV positivity, defined by either EBER cut-off ( $20 \%$ or $50 \%)$, had a close association with male sex, advanced clinical stage, poor PS status and lower response to first-line treatment, which was observed in both young and elderly patients. These results indicated that EBV positive DLBCL were clinically aggressive, irrespective of age. Based on these, we then analyzed the association between EBV positivity and the clinical prognostic indicators reported previously ${ }^{27-32}$. The results showed that EBV positivity had a strong relationship with elevated serum CRP level, $\beta 2 \mathrm{M}$ level, CA125 level, ESR level and ferritin level, regardless of age and EBER cut-off value. 


\begin{tabular}{|c|c|c|c|c|c|c|c|c|c|c|}
\hline \multirow[b]{3}{*}{ Variable } & \multirow{3}{*}{$\begin{array}{c}\text { Number. } \\
(\%)\end{array}$} & \multicolumn{2}{|c|}{ All age } & \multirow[b]{3}{*}{$P$ value } & \multicolumn{2}{|c|}{ Elderly group } & \multirow[b]{3}{*}{$P$ value } & \multicolumn{2}{|c|}{ Young group } & \multirow{3}{*}{$\begin{array}{c}P \\
\text { value }\end{array}$} \\
\hline & & EBV+ & EBV- & & EBV+ & EBV- & & EBV+ & EBV- & \\
\hline & & \multicolumn{2}{|c|}{ Number.(\%) } & & \multicolumn{2}{|c|}{ Number.(\%) } & & \multicolumn{2}{|c|}{ Number.(\%) } & \\
\hline CD10 & 250 & 26 & 224 & & 19 & 147 & & 7 & 77 & \\
\hline Positive & $60(24.0)$ & $3(11.5)$ & $57(25.4)$ & 0.116 & $3(18.6)$ & $36(24.5)$ & 0.568 & $0(0.0)$ & $21(27.3)$ & 0.184 \\
\hline Negative & $190(76.0)$ & $23(88.5)$ & $167(74.6)$ & & $16(81.2)$ & $111(75.5)$ & & $7(100.0)$ & $56(72.7)$ & \\
\hline Bcl6 & 248 & 26 & 222 & & 19 & 146 & & 7 & 76 & \\
\hline Positive & $160(64.5)$ & $6(23.1)$ & $154(69.4)$ & $<0.001$ & $4(21.1)$ & $94(64.4)$ & $<0.001$ & $2(28.6)$ & $60(78.9)$ & 0.010 \\
\hline Negative & $88(35.5)$ & $20(76.9)$ & $68(30.6)$ & & $15(78.9)$ & $52(35.6)$ & & $5(71.4)$ & $16(21.1)$ & \\
\hline MUM1 & 250 & 26 & 224 & & 19 & 147 & & 7 & 77 & \\
\hline Positive & $157(62.8)$ & $15(57.7)$ & $142(63.4)$ & 0.569 & $11(57.9)$ & $100(68.0)$ & 0.377 & $4(57.1)$ & $42(54.5)$ & 1.000 \\
\hline Negative & $93(37.2)$ & $11(42.3)$ & $82(36.6)$ & & $8(42.1)$ & $47(32.0)$ & & $3(42.9)$ & $35(45.5)$ & \\
\hline GCET1 & 245 & 23 & 222 & & 16 & 147 & & 7 & 75 & \\
\hline$\geq 60 \%$ & $31(12.7)$ & $1(4.3)$ & $30(13.5)$ & 0.326 & $0(0.0)$ & $20(13.6)$ & 0.223 & $1(14.3)$ & $10(13.3)$ & 1.000 \\
\hline$<60 \%$ & $214(87.3)$ & $22(95.7)$ & $192(86.5)$ & & $16(100.0)$ & $127(86.4)$ & & $6(85.7)$ & $65(86.7)$ & \\
\hline FOXP1 & 230 & 23 & 207 & & 16 & 136 & & 7 & 71 & \\
\hline$\geq 60 \%$ & $138(60.1)$ & $10(43.5)$ & $128(61.8)$ & 0.088 & $6(37.5)$ & 93 (68.4) & 0.014 & $4(57.1)$ & $35(49.3)$ & 1.000 \\
\hline$<60 \%$ & $92(35.9)$ & $13(56.5)$ & $79(38.2)$ & & $10(62.5)$ & $43(31.6)$ & & $3(42.9)$ & $36(50.7)$ & \\
\hline $\mathrm{LMO} 2$ & 244 & 23 & 221 & & 16 & 146 & & 7 & 75 & \\
\hline$\geq 30 \%$ & $194(79.5)$ & 17 (73.9) & $177(80.1)$ & 0.586 & $14(87.5)$ & $112(76.7)$ & 0.527 & $3(42.9)$ & $65(86.7)$ & 0.014 \\
\hline$<30 \%$ & $50(20.5)$ & $6(26.1)$ & 44 (19.9) & & $2(12.5)$ & $34(26.3)$ & & $4(57.1)$ & $10(13.3)$ & \\
\hline CD5 & 250 & 26 & 224 & & 19 & 147 & & 7 & 77 & \\
\hline Positive & $17(6.8)$ & $2(7.7)$ & $15(6.7)$ & 1.000 & $1(5.3)$ & $7(4.8)$ & 1.000 & $1(14.3)$ & $8(10.4)$ & 1.000 \\
\hline Negative & $233(93.2)$ & $24(92.3)$ & $209(93.3)$ & & $18(94.7)$ & $140(95.2)$ & & $6(85.7)$ & 69 (89.6) & \\
\hline CD30 & 250 & 26 & 224 & & 19 & 147 & & 7 & 77 & \\
\hline Positive & $25(10 \%)$ & $10(38.5)$ & $15(6.7)$ & $<0.001$ & $7(36.8)$ & $9(6.1)$ & $<0.001$ & $3(42.9)$ & $6(7.8)$ & 0.024 \\
\hline Negative & $225(90.0)$ & $16(61.5)$ & $209(93.3)$ & & $12(63.2)$ & $138(93.9)$ & & $4(57.1)$ & $71(92.2)$ & \\
\hline Myc & 248 & 24 & 224 & & 17 & 147 & & 7 & 77 & \\
\hline Positive & 91 (36.7) & $12(50.0)$ & 79 (35.3) & 0.155 & $9(52.9)$ & $58(39.5)$ & 0.284 & $3(42.9)$ & $21(27.3)$ & 0.402 \\
\hline Negative & $157(63.3)$ & $12(50.0)$ & $145(64.7)$ & & $8(47.1)$ & $89(60.5)$ & & $4(57.1)$ & $56(72.7)$ & \\
\hline $\mathrm{Bcl} 2$ & 249 & 26 & 223 & & 19 & 146 & & 7 & 77 & \\
\hline Positive & $142(57.0)$ & $8(30.8)$ & $134(60.1)$ & 0.004 & $6(31.6)$ & $91(62.3)$ & 0.010 & $2(28.6)$ & $43(55.8)$ & 0.242 \\
\hline Negative & $107(43.0)$ & 18 (69.2) & 89 (39.9) & & $13(8.4)$ & $55(37.7)$ & & $5(71.4)$ & $34(44.2)$ & \\
\hline DPE & $64(25.6)$ & $8(30.8)$ & $56(25.0)$ & 0.523 & $6(31.6)$ & $40(27.2)$ & 0.689 & $2(28.6)$ & $16(20.8)$ & 0.639 \\
\hline Others & $186(74.4)$ & $18(69.2)$ & $168(75.0)$ & & $13(68.4)$ & $107(72.8)$ & & $5(71.4)$ & $61(79.2)$ & \\
\hline Ki-67 & 249 & 25 & 224 & & 18 & 147 & & 7 & 77 & \\
\hline$\geq 70 \%$ & 97 (39.0) & $17(68.0)$ & $80(35.7)$ & $<0.001$ & $12(66.7)$ & $53(36.1)$ & 0.001 & $5(71.4)$ & $27(35.1)$ & 0.100 \\
\hline$<70 \%$ & $152(61.0)$ & $8(32.0)$ & $144(64.3)$ & & $6(33.3)$ & $94(63.9)$ & & $2(28.6)$ & $50(64.9)$ & \\
\hline MYC-ba & 246 & 23 & 223 & & 17 & 146 & & 6 & 77 & \\
\hline Positive & $33(13.4)$ & $6(26.1)$ & $27(12.1)$ & 0.099 & $4(23.5)$ & $18(12.3)$ & 0.252 & $2(33.3)$ & $9(11.7)$ & 0.178 \\
\hline Negative & $213(86.6)$ & $17(73.9)$ & $196(87.9)$ & & $13(76.5)$ & $128(87.7)$ & & $4(66.7)$ & $68(88.3)$ & \\
\hline BCL2/IGH & 247 & 23 & 224 & & 17 & 146 & & 6 & 77 & \\
\hline Positive & $32(13.0)$ & $6(26.1)$ & 26 (11.6) & 0.170 & $3(17.6)$ & $20(13.7)$ & 0.711 & $3(50.0)$ & $6(7.8)$ & 0.015 \\
\hline Negative & $215(87.0)$ & $17(73.9)$ & $198(88.4)$ & & $14(82.4)$ & $127(86.3)$ & & $3(50.0)$ & $71(92.2)$ & \\
\hline DHL & $6(2.4)$ & $3(12.5)$ & $3(1.3)$ & 0.013 & $2(11.8)$ & $2(1.4)$ & 0.054 & $1(14.3)$ & $1(1.3)$ & 0.161 \\
\hline Non-DHL & 242 (97.6) & $21(87.5)$ & $221(98.7)$ & & $15(88.2)$ & 145 (98.6) & & $6(85.7)$ & 76 (98.7) & \\
\hline Hans & 250 & 26 & 224 & & 19 & 147 & & 7 & 77 & \\
\hline GCB & $98(39.2)$ & $10(38.5)$ & $88(39.3)$ & 0.139 & $8(42.1)$ & $50(34.0)$ & 0.486 & $2(28.6)$ & $38(49.4)$ & 0.437 \\
\hline Non-GCB & $152(60.8)$ & $16(61.5)$ & $136(60.7)$ & & $11(57.9)$ & $97(66.0)$ & & $5(71.4)$ & $39(50.6)$ & \\
\hline
\end{tabular}




\begin{tabular}{|c|c|c|c|c|c|c|c|c|c|c|}
\hline \multirow[b]{3}{*}{ Variable } & \multirow{3}{*}{$\underset{(\%)}{\text { Number. }}$} & \multicolumn{2}{|c|}{ All age } & \multirow[b]{3}{*}{$P$ value } & \multicolumn{2}{|c|}{ Elderly group } & \multirow[b]{3}{*}{$P$ value } & \multicolumn{2}{|c|}{ Young group } & \multirow{3}{*}{$\begin{array}{c}P \\
\text { value }\end{array}$} \\
\hline & & EBV+ & EBV- & & EBV+ & EBV- & & EBV+ & EBV- & \\
\hline & & \multicolumn{2}{|c|}{ Number.(\%) } & & \multicolumn{2}{|c|}{ Number.(\%) } & & \multicolumn{2}{|c|}{ Number.(\%) } & \\
\hline Choi & 244 & 23 & 221 & & 16 & 146 & & 7 & 75 & \\
\hline GCB & $110(45.1)$ & $6(26.1)$ & $104(47.1)$ & 0.054 & $5(31.2)$ & $60(41.1)$ & 0.446 & $1(14.3)$ & $44(58.6)$ & 0.042 \\
\hline Non-GCB & $134(54.9)$ & $17(73.9)$ & $117(52.9)$ & & $11(68.8)$ & $86(58.9)$ & & $6(85.7)$ & $31(41.3)$ & \\
\hline Tally & 246 & 23 & 223 & & 16 & 147 & & 7 & 76 & \\
\hline GCB & $68(27.6)$ & $7(30.4)$ & $61(27.4)$ & 0.422 & $6(37.5)$ & $36(24.5)$ & 0.105 & $1(14.3)$ & $25(32.9)$ & 0.425 \\
\hline Non-GCB & $178(72.4)$ & $16(69.6)$ & $162(72.6)$ & & $10(62.5)$ & $111(75.5)$ & & $6(85.7)$ & $51(67.1)$ & \\
\hline Visco-Young & 246 & 25 & 221 & & 18 & 146 & & 7 & 75 & \\
\hline GCB & $106(43.1)$ & $7(28.0)$ & $99(44.8)$ & 0.108 & $5(27.8)$ & $58(39.7)$ & 0.325 & $2(28.6)$ & $41(54.7)$ & 0.249 \\
\hline Non-GCB & $140(56.9)$ & $18(72.0)$ & $122(55.2)$ & & $13(72.2)$ & $88(60.3)$ & & $5(71.4)$ & $34(45.3)$ & \\
\hline
\end{tabular}

Table 6. Pathological features of patients in different groups (EBER cut-off: 50\%). Abbreviations: EBV: Epstein-Barr virus; DPE: double protein expression; ba: break apart; DHL: double hit lymphoma; GCB:germinal center B cell.
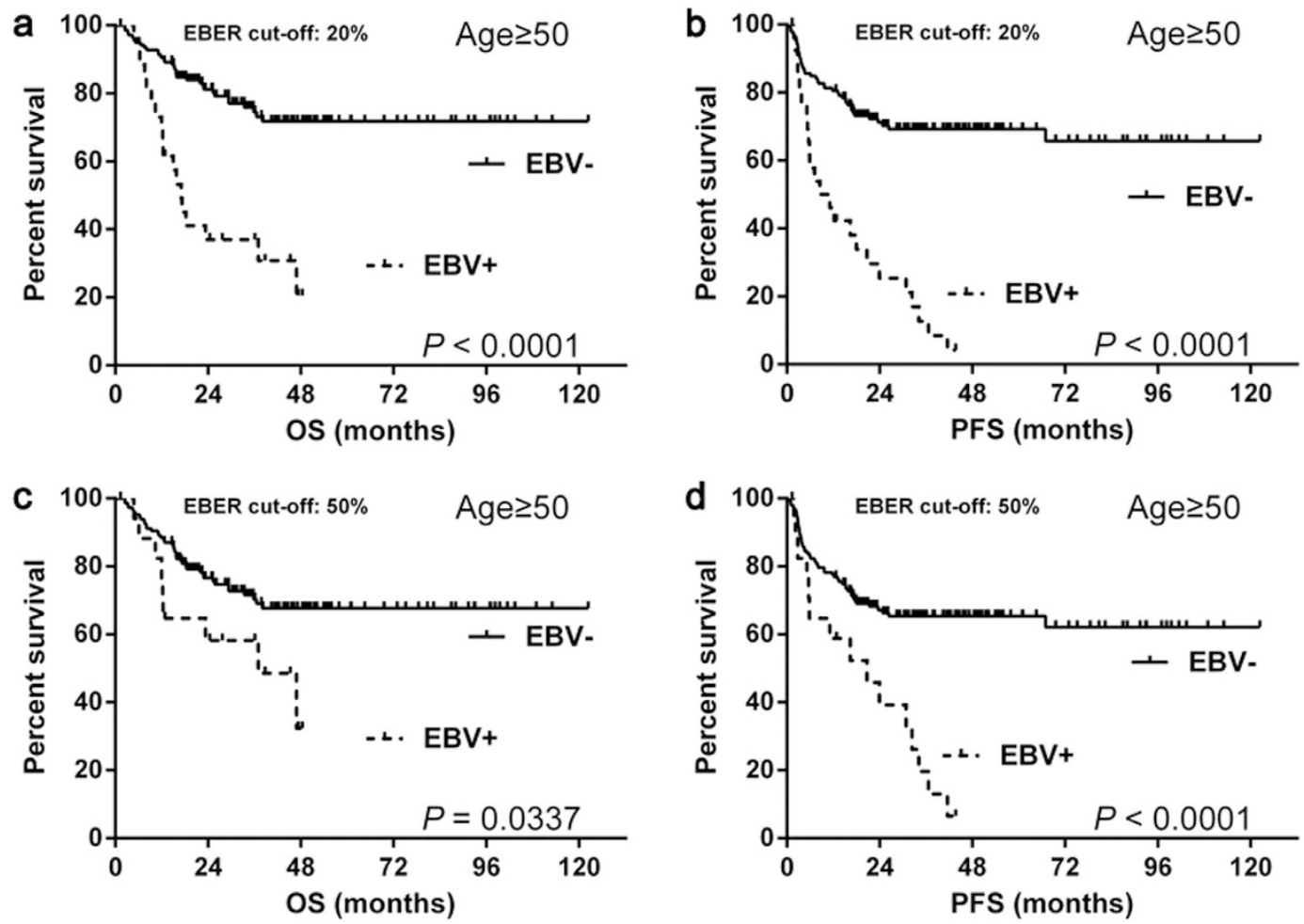

Figure 2. The survival differences of elderly DLBCL with EBV status. The EBV positive patients of the elderly group showed significantly worse OS $(\mathbf{a}, \mathbf{c})$ and PFS (b,d) than negative ones, regardless of the EBER cut-off values. Abbreviations: EBER: Epstein-Barr virus-encoded RNA; EBV: Epstein-Barr virus; OS: overall survival; PFS: progression-free survival.

We subsequently included CD10, Bcl6, MUM1, FOXP1, GCET1 and LMO2 in our study as these markers were used to establish a diagnosis and further classify DLCBL into GCB or non-GCB subtype ${ }^{21-24}$. Research showed the majority of EBV positive DLBCL of the elderly had a non-GCB predominance, which is a subtype with poor prognosis. In our study, we accessed the COO with four conventional IHC algorithms. Although EBV positive DLBCL of the elderly group showed less frequent FOXP1 expression and the young group demonstrated less common of LMO2 expression, the EBV positive DLBCL (both age groups), although not statistically significant, demonstrated the non-GCB subtype preference than negative ones. However, the COO difference was observed in Choi and Visco-Young algorithms alone. The poor concordance among IHC algorithms may be one of reasons to explain our 

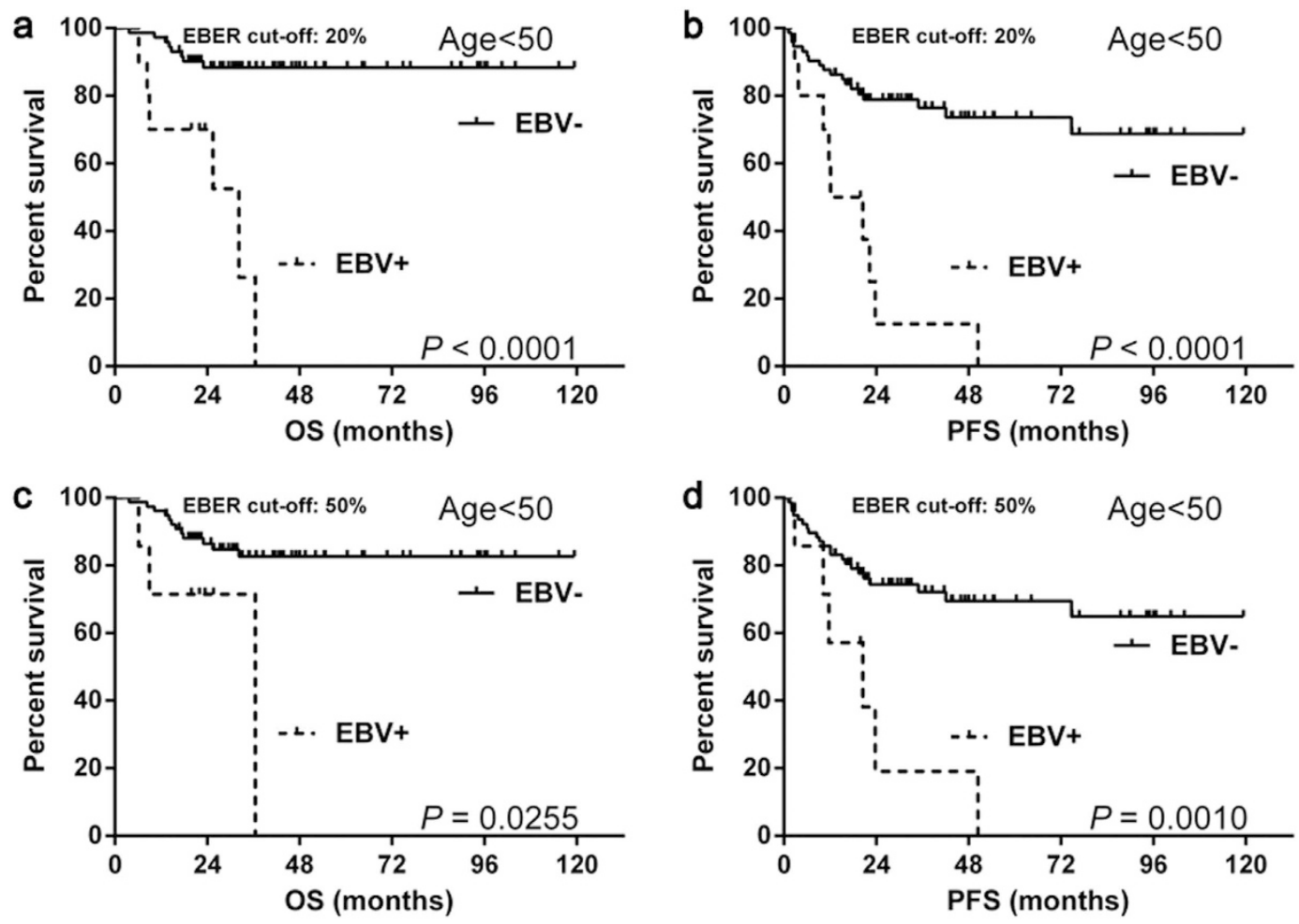

Figure 3. The survival differences of young DLBCL with EBV status. The EBV positive patients of the young group showed significantly worse OS $(\mathbf{a}, \mathbf{c})$ and PFS $(\mathbf{b}, \mathbf{d})$ than negative ones, regardless of the EBER cut-off values. Abbreviations: EBER: Epstein-Barr virus-encoded RNA; EBV: Epstein-Barr virus; OS: overall survival; PFS: progression-free survival.

results, since $20-30 \%$ cases among the IHC algorithms are discrepant ${ }^{22,33}$. We also analyzed CD5, Myc, $\mathrm{Bcl} 2, \mathrm{Ki}-67$ expression and DPE, MYC and BCL2 gene rearrangement and DHL because of their efficacy in prognostication ${ }^{34-36}$. We also chose CD30 due its significant association with EBV positivity in DLBCL that was observed in several previous studies ${ }^{7,15,37}$. Moreover, EBER ${ }^{+} / \mathrm{CD} 0^{+}$DLBCL had significantly poorer outcomes compared with $\mathrm{EBER}^{-} / \mathrm{CD} 30^{+}$cases $^{38}$. Using the cut-off values of these proteins applied in above studies $7,34-37$, we accessed the incidences of these pathological factors in DLBCL according to EBV status and age. Interestingly, EBER positivity was associated with less frequent Bcl6 expression, which was also confirmed by previous studies ${ }^{7}$. Moreover, Constitutive expression of EBV-derived miRNAs, including BART3, BART7, BART9 and BART17-5p, was found to be capable of repressing Bcl6 expression, partly accounting for reduced Bcl6 expression in EBV positive lymphoma ${ }^{39}$. The proportions of Myc expression, DPE, MYC rearrangement and DHL were much higher in EBV positive DLBCL, which were consistent with the aggressive biological behavior of EBV positive DLBCL ${ }^{1,11,13}$. DPE and DHL were more frequent in EBV positive cases than EBV negative ones, which was inconsistent with the study by Ok et al. ${ }^{7}$, probably due to different ethnic background or geographic variation of EBV strains. $\mathrm{Bcl} 2$ expression was less frequent in EBV positive DLBCL. However, this was not observed in a previous study $^{7}$. Of note, in that study, cut-off for $\mathrm{Bcl} 2$ was $70 \%$, rather than $50 \%$. Higher Ki-67 expression was observed in EBV positive patients, suggesting that EBV infection contributes the aggressiveness of EBV positive DLBCL ${ }^{40}$. Similar to previous studies ${ }^{40}$, the significant association of CD30 with EBV positivity in DLBCL was also confirmed in our study. However, the mechanism underlying this phenomenon remains to be determined. It is possible that CD30 functions synergistically with EBV to transform B lymphocytes ${ }^{41}$.

In present study, although the treatment for EBV positive DLBCL was more intensive than negative ones (much higher percentage of immunochemotherapy in EBV positive DLBCL), consistent with most studies $^{4,12,16,19}$, both age groups with EBV positive DLBCL showed significantly worse OS and PFS than negative cases. Further comparison showed that EBV positive DLBCL of the elderly group had similar OS and PFS to the young counterparts. It is worth mentioning that the EBV positive DLBCL patients (both elderly and young) were treated with similar regimens. Actually, EBV positive DLBCL of the elderly was included as a provisional entity in the 2008 WHO classification of tumors of hematopoietic and lymphoid tissues, in which the WHO Working Group considered that there was insufficient evidence to recognize this entity as a distinct disease at that time ${ }^{3,42}$. EBV positive DLBCL of the young group that had no evidence of underlying immunosuppression had been described in previous reports ${ }^{4,16}$. The identification 

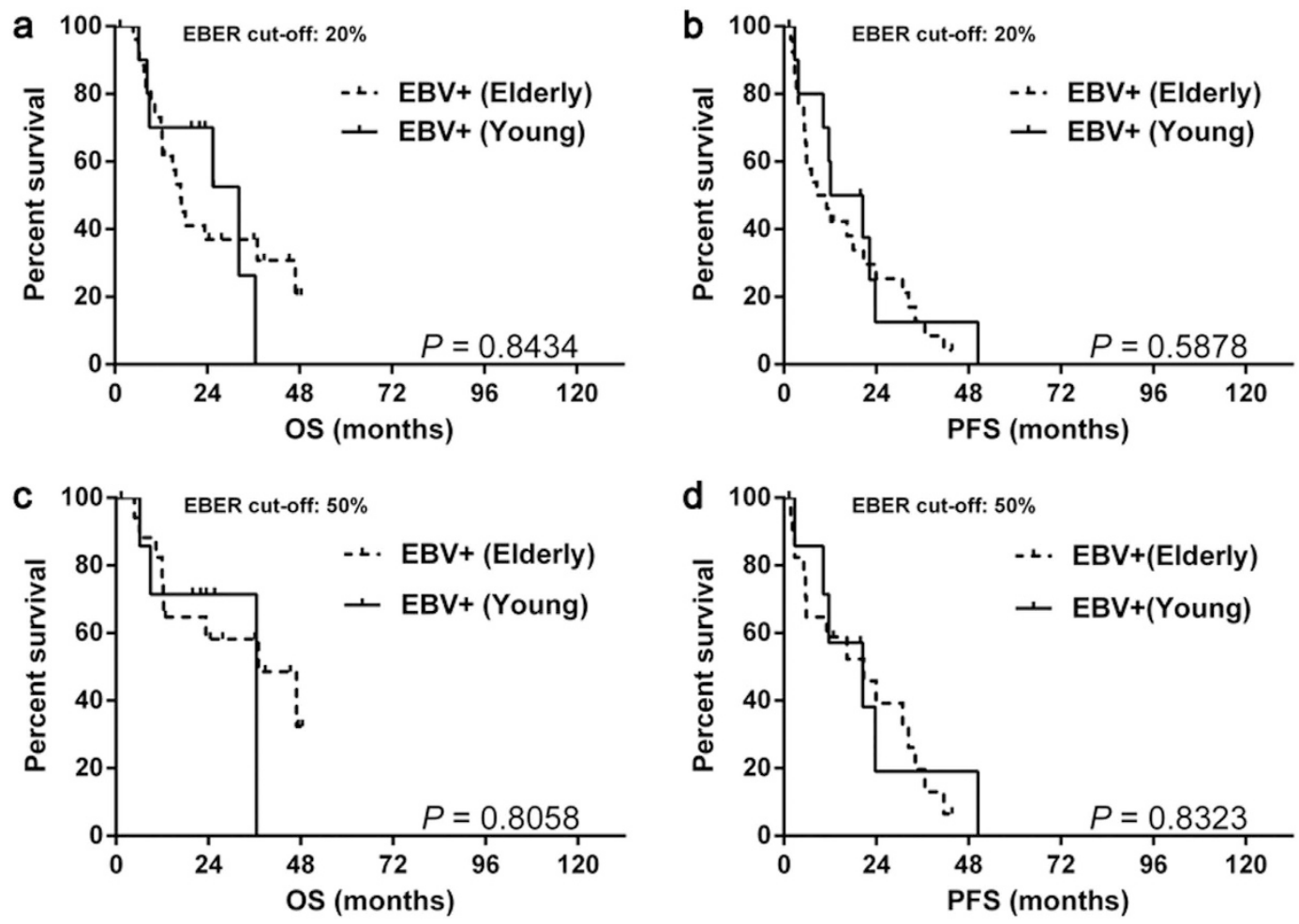

Figure 4. The survival differences of EBV positive DLBCL beween different age groups. The EBV positive DLBCL of the elderly group showed similar OS (a,c) and PFS (b,d) with the young ones, regardless of the EBER cut-off values. Abbreviations: EBER: Epstein-Barr virus-encoded RNA; EBV: Epstein-Barr virus; OS: overall survival; PFS: progression-free survival.

of these young cases arised an interrogation that if EBV positive DLBCL might be an entity that was not restricted to patients who were older than 50 years old alone. However, one recent study did not recognized unfavorable baseline characteristics in young patients with EBV positive DLBCL ${ }^{14}$. Therefore, they concluded that EBV positive DLBCL of young group should be considered to be a distinct clinical entity different from EBV positive DLBCL of the elderly ${ }^{14}$. In present study, to say the least, no distinct difference was recognized in the clinical and pathological features between EBV positive DLBCL in the elderly and the young group, which was in accordance with most studies ${ }^{4,12}$. These unfavorable factors were shared by both groups, which indicated that EBV positive DLBCL belongs to a unique entity with aggressive clinical features $7,11,13-15,20,43$, regardless of age. Since there is no uniform cut-off for EBV positivity, some reports attributed the inconsistent results to the different cut-off values used in different studies $^{7,11,13,14,44-46}$. However, by appling two most frequently-used cut-off values, our study indicated that EBER positive patients harbored more unfavorable clinical and pathological features than EBER negative ones, regardless of the cut-off values of EBV.

Several studies had reported that EBV positive patients showed an inferior prognosis compared with EBV negative cases, especially the elderly group ${ }^{7,11,13,15,18,47,48}$. The clinical course is often aggressive with a median survival of 2 years and an overall 5-year survival rate of approximately $25 \%{ }^{4,5,8}$. In our study, accordantly, EBV positive DLBCL of the elderly showed worse survival than negative counterparts. Many studies also showed the young patients of EBV positivity demonstrated poor outcome with traditional immunochemotherapy $4,12,16,19$, which was confirmed in current study. However, the study by Hong et al. revealed young EBV positive DLBCL patients $(6.7 \%, 13 / 195)$ had an outcome similar to EBV negative ones $^{14}$.

In fact, the biological mechanism underlying similar clinical and pathological features and outcome between these age groups remains to be investigated. To elucidate molecular mechanisms involved EBV positive DLBCL, Ok et al. ${ }^{7}$ evaluated GEP profiles signatures of DLBCL with different EBV status. It is worth noting that in the $24 \mathrm{EBV}$ positive patients included in that study, 7 patients were younger than 50 years old. They revealed the NF- $\kappa B$ activation and JAK/STAT activation were enhanced in EBV positive DLBCL compared with negative counterparts by gene set enrichment analysis. Thus, EBV positive DLBCL might share similar GEP signatures and common pathogenic pathways, irrespective of age. It also has been reported that ABC-like DLBCL more frequently exhibited JAK-STAT and NF- $\kappa$ B pathways activation compared with GCB-like DLBCL ${ }^{49,50}$ Interestingly, Kato et al. demonstrated that NF- $\kappa \mathrm{B}$ and JAK-STAT pathways were more remarkably activated in EBV positive DLBCL compared with ABC-like 
EBV negative DLBCL ${ }^{47}$. Using miRNA array platforms, Andrade et al. ${ }^{43}$ showed that miR-146b and miR-222 were highly specific for EBV positive DLBCL. The targets of hsa-miR-146b and its viral counterpart are INTS6 and IPO, both of which are tumor suppressors ${ }^{51}$ and mediators of inflammation ${ }^{26}$ Similarly, hsa-miR-222 interferes with important proteins related to oncogenesis, cell cycle regulation, cell transcription, cell adhesion, oxidative stress, and apoptosis inhibition ${ }^{52}$. Besides, NF- $\kappa B$ pathway can be activated indirectly by hsa-miR-222 ${ }^{43}$. All of the above findings suggested that EBV positive DLBCL shared a common molecular basis, irrespective of age.

Additionally, using other age cut-off values, we did not recognized any significant differences of survival between elderly and the young patients of EBV positivity. This also indicated that the young group patients should not be excluded from the whole cohort of EBV positive patients and the 50 years old cut-off might not be applicable in the R-CHOP era.

In summary, EBV positive DLBCL patients shared poor prognostic features, regardless of elderly or young group. The survival analysis also showed that EBV DLBCL of the elderly showed a similar outcome to the young ones. Based on these results, we suggest that the age criterion, and possibly the name-EBV positive DLBCL of elderly itself, be modified in future.

\section{References}

1. Jaffe, E. S. \& Pittaluga, S. Aggressive B-cell lymphomas: a review of new and old entities in the WHO classification. Hematology Am Soc Hematol Educ Program 2011, 506-514 (2011).

2. Campo, E. et al. The 2008 WHO classification of lymphoid neoplasms and beyond: evolving concepts and practical applications. Blood 117, 5019-5032 (2011).

3. Swerdlow, S. H. et al. WHO Classification of Tumours of Hematopoietic and Lymphoid Tissues. 4th ed. Lyon: IARC, (2008).

4. Park, S. et al. The impact of Epstein-Barr virus status on clinical outcome in diffuse large B-cell lymphoma. Blood 110, 972-978 (2007).

5. Oyama, T. et al. Age-related EBV-associated B-cell lymphoproliferative disorders constitute a distinct clinicopathologic group: a study of 96 patients. Clin Cancer Res 13, 5124-5132 (2007).

6. Kuze, T. et al. The characteristics of Epstein-Barr virus (EBV)-positive diffuse large B-cell lymphoma: comparison between $\mathrm{EBV}(+)$ and EBV(-) cases in Japanese population. Jpn J Cancer Res 91, 1233-1240 (2000).

7. Ok, C. Y. et al. Prevalence and clinical implications of epstein-barr virus infection in de novo diffuse large B-cell lymphoma in Western countries. Clin Cancer Res 20, 2338-2349 (2014).

8. Gibson, S. E. \& Hsi, E. D. Epstein-Barr virus-positive B-cell lymphoma of the elderly at a United States tertiary medical center: an uncommon aggressive lymphoma with a nongerminal center B-cell phenotype. Hum Pathol 40, 653-661 (2009).

9. Coiffier, B. et al. Long-term outcome of patients in the LNH-98.5 trial, the first randomized study comparing rituximab-CHOP to standard CHOP chemotherapy in DLBCL patients: a study by the Groupe d'Etudes des Lymphomes de l'Adulte. Blood 116, 2040-2045 (2010).

10. Feugier, P. et al. Long-term results of the R-CHOP study in the treatment of elderly patients with diffuse large B-cell lymphoma: a study by the Groupe d'Etude des Lymphomes de l'Adulte. J Clin Oncol 23, 4117-4126 (2005).

11. Ahn, J. S. et al. Clinical outcome of elderly patients with Epstein-Barr virus positive diffuse large B-cell lymphoma treated with a combination of rituximab and CHOP chemotherapy. Am J Hematol 88, 774-779 (2013).

12. Morales, D. et al. Epstein-Barr virus as a prognostic factor in de novo nodal diffuse large B-cell lymphoma. Leuk Lymphoma 51, 66-72 (2010).

13. Sato, A. et al. Clinical outcome of Epstein-Barr virus-positive diffuse large B-cell lymphoma of the elderly in the rituximab era. Cancer Sci 105, 1170-1175 (2014).

14. Hong, J. Y. et al. EBV-positive diffuse large B-cell lymphoma in young adults: is this a distinct disease entity? Ann Oncol 26, 548-555 (2014)

15. Montes-Moreno, S. et al. EBV-positive diffuse large B-cell lymphoma of the elderly is an aggressive post-germinal center B-cell neoplasm characterized by prominent nuclear factor-kB activation. Mod Pathol 25, 968-982 (2012).

16. Beltran, B. E. et al. EBV-positive diffuse large b-cell lymphoma in young immunocompetent individuals. Clin Lymphoma Myeloma Leuk 11, 512-516 (2011).

17. Cohen, M. et al. Epstein-Barr virus-positive diffuse large B-cell lymphoma association is not only restricted to elderly patients. Int J Cancer 135, 2816-2824 (2014).

18. Hofscheier, A. et al. Geographic variation in the prevalence of Epstein-Barr virus-positive diffuse large B-cell lymphoma of the elderly: a comparative analysis of a Mexican and a German population. Mod Pathol 24, 1046-1054 (2011).

19. Castillo, J. J. et al. Epstein-barr virus-positive diffuse large B-cell lymphoma of the elderly: what we know so far. Oncologist 16, 87-96 (2011)

20. Ok, C. Y. et al. EBV-positive diffuse large B-cell lymphoma of the elderly. Blood 122, 328-340 (2013).

21. Visco, C. et al. Comprehensive gene expression profiling and immunohistochemical studies support application of immunophenotypic algorithm for molecular subtype classification in diffuse large B-cell lymphoma: a report from the International DLBCL RituximabCHOP Consortium Program Study. Leukemia 26, 2103-2113 (2012).

22. Meyer, P. N. et al. Immunohistochemical methods for predicting cell of origin and survival in patients with diffuse large B-cell lymphoma treated with rituximab. J Clin Oncol 29, 200-207 (2011).

23. Choi, W. W. et al. A new immunostain algorithm classifies diffuse large B-cell lymphoma into molecular subtypes with high accuracy. Clin Cancer Res 15, 5494-5502 (2009).

24. Hans, C. P. et al. Confirmation of the molecular classification of diffuse large B-cell lymphoma by immunohistochemistry using a tissue microarray. Blood 103, 275-282 (2004).

25. Cheson, B. D. et al. Recommendations for initial evaluation, staging, and response assessment of Hodgkin and non-Hodgkin lymphoma: the Lugano classification. J Clin Oncol 32, 3059-3068 (2014).

26. Beltran, B. E. et al. EBV-positive diffuse large B-cell lymphoma of the elderly: a case series from Peru. Am J Hematol 86, 663-667 (2011).

27. Adams, H. J. et al. False-negative FDG-PET in histologically proven extensive large cell bone marrow involvement in diffuse large B-cell lymphoma. Am J Hematol 90, doi: 10.1002/ajh.23986 (2015).

28. Troppan, K. T. et al. C-reactive protein level is a prognostic indicator for survival and improves the predictive ability of the R-IPI score in diffuse large B-cell lymphoma patients. Br J Cancer 111, 55-60 (2014).

29. Tisi, M. C. et al. Anemia in diffuse large B-cell non-Hodgkin lymphoma: the role of interleukin-6, hepcidin and erythropoietin. Leuk Lymphoma 55, 270-275 (2014). 
30. Duletic-Nacinovic, A. et al. Serum IL-6, IL-8, IL-10 and beta2-microglobulin in association with International Prognostic Index in diffuse large B cell lymphoma. Tumori 94, 511-517 (2008).

31. Vlasveld, L. T. et al. Elevated serum CA-125 concentrations due to expression by a diffuse large B-cell lymphoma. Ann Clin Biochem 37 (Pt 4), 545-548 (2000).

32. Nicolaides, C. et al. Diffuse large cell lymphomas: identification of prognostic factors and validation of the International NonHodgkin's Lymphoma Prognostic Index. A Hellenic Cooperative Oncology Group Study. Oncology 55, 405-415 (1998).

33. Coutinho, R. et al. Poor concordance among nine immunohistochemistry classifiers of cell-of-origin for diffuse large B-cell lymphoma: implications for therapeutic strategies. Clin Cancer Res 19, 6686-6695 (2013).

34. Hu, S. et al. MYC/BCL2 protein coexpression contributes to the inferior survival of activated B-cell subtype of diffuse large B-cell lymphoma and demonstrates high-risk gene expression signatures: a report from The International DLBCL Rituximab-CHOP Consortium Program. Blood 121, 4021-4031 (2013).

35. Yoon, D. H. et al. Ki-67 expression as a prognostic factor in diffuse large B-cell lymphoma patients treated with rituximab plus CHOP. Eur J Haematol 85, 149-157 (2010).

36. Miyazaki, K. et al. CD5-positive diffuse large B-cell lymphoma: a retrospective study in 337 patients treated by chemotherapy with or without rituximab. Ann Oncol 22, 1601-1607 (2011).

37. Slack, G. W. et al. CD30 expression in de novo diffuse large B-cell lymphoma: a population-based study from British Columbia. Br J Haematol 167, 608-617 (2014).

38. Hu, S. et al. CD30 expression defines a novel subgroup of diffuse large B-cell lymphoma with favorable prognosis and distinct gene expression signature: a report from the International DLBCL Rituximab-CHOP Consortium Program Study. Blood 121, 2715-2724 (2013).

39. Martin-Perez, D. et al. Epstein-Barr virus microRNAs repress BCL6 expression in diffuse large B-cell lymphoma. Leukemia 26, 180-183 (2012).

40. Wong, H. H., Wang, J. Epstein-Barr virus positive diffuse large B-cell lymphoma of the elderly. Leuk Lymphoma 50, 335-340 (2009).

41. Vockerodt, M. et al. The Epstein-Barr virus and the pathogenesis of lymphoma. J Pathol 235, 312-322 (2015).

42. Zelenetz., A. D. et al. NCCN Clinical Practice Guidelines in Oncology. Non-Hodgkin's Lymphomas. Version 1, 160-161 (2015).

43. Andrade, T. A. et al. A microRNA signature profile in EBV+ diffuse large B-cell lymphoma of the elderly. Oncotarget 5, 11813$11826(2014)$

44. Ozsan, N. et al. Epstein-Barr virus (EBV) positive diffuse large B cell lymphoma of the elderly-experience of a single center from Turkey. Pathol Res Pract 209, 471-478 (2013).

45. Morscio, J. et al. Gene expression profiling reveals clear differences between EBV-positive and EBV-negative posttransplant lymphoproliferative disorders. Am J Transplant 13, 1305-1316 (2013).

46. Wada, N. et al. Epstein-barr virus in diffuse large B-Cell lymphoma in immunocompetent patients in Japan is as low as in Western Countries. J Med Virol 83, 317-321 (2011).

47. Kato, H. et al. Gene expression profiling of Epstein-Barr virus-positive diffuse large B-cell lymphoma of the elderly reveals alterations of characteristic oncogenetic pathways. Cancer Sci 105, 537-544 (2014).

48. De Unamuno Bustos, B. et al. Epstein-Barr virus-positive diffuse large B-cell lymphoma in an elderly patient. Clin Exp Dermatol 39, 484-487 (2014).

49. Scuto, A. et al. STAT3 inhibition is a therapeutic strategy for ABC-like diffuse large B-cell lymphoma. Cancer Res 71, 3182-3188 (2011).

50. Lam, L. T. et al. Cooperative signaling through the signal transducer and activator of transcription 3 and nuclear factor-\{kappa\} B pathways in subtypes of diffuse large B-cell lymphoma. Blood 111, 3701-3713 (2008).

51. Lei, T. et al. Targeting of DICE1 tumor suppressor by Epstein-Barr virus-encoded miR-BART3* microRNA in nasopharyngeal carcinoma. Int J Cancer 133, 79-87 (2013).

52. Jansson, M. D. \& Lund, A. H. MicroRNA and cancer. Mol Oncol 6, 590-610 (2012).

\section{Acknowledgments}

This study was supported by National Natural Science Foundation of China $(30971296,81170485$, 81170488, 81370657, 81470328), Key Projects of Health Department of Jiangsu Province (K201108), Jiangsu Province's Medical Elite Program (RC2011169), National Public Health Grand Research Foundation (201202017), Project Funded by the Priority Academic Program Development of Jiangsu Higher Education Institute (JX10231801), Program for Development of Innovative Research Teams in the First Affiliated Hospital of Nanjing Medical University, Project of National Key Clinical Specialty. National Science \& Technology Pillar Program (2014BAI09B12), and Project funded by Jiangsu Provincial Special Program of Medical Science (BL2014086).

\section{Author Contributions}

T.L., Z.Z., W.X. and J.L. designed the experiment. T.L., Q.G. and Z.W. performed the experiments. T.L., J.L., Y.M., L.F., L.W., X.Q. and L.C. organized the clinical materials. T.L. and Y.M. perfomed the analysis. T.L. and Y.M. wrote the paper.

\section{Additional Information}

Competing financial interests: The authors declare no competing financial interests.

How to cite this article: Lu, T.-X. et al. Epstein-Barr virus positive diffuse large B-cell lymphoma predict poor outcome, regardless of the age. Sci. Rep. 5, 12168; doi: 10.1038/srep12168 (2015).

This work is licensed under a Creative Commons Attribution 4.0 International License. The images or other third party material in this article are included in the article's Creative Commons license, unless indicated otherwise in the credit line; if the material is not included under the Creative Commons license, users will need to obtain permission from the license holder to reproduce the material. To view a copy of this license, visit http://creativecommons.org/licenses/by/4.0/ 